\title{
Splittings of free groups, normal forms and partitions of ends
}

\author{
SIDDHARTHA GADGIL ${ }^{1}$ and SUHAS PANDIT ${ }^{2}$ \\ ${ }^{1}$ Department of Mathematics, Indian Institute of Science, Bangalore 560 012, India \\ ${ }^{2}$ Department of Mathematics, Indian Institute of Science Education and Research, \\ Pune 411 021, India \\ E-mail: gadgil@math.iisc.ernet.in; suhasjp@iiser.ac.in
}

MS received 5 June 2009; revised 21 January 2010

\begin{abstract}
Splittings of a free group correspond to embedded spheres in the 3-manifold $M=\sharp_{k} S^{2} \times S^{1}$. These can be represented in a normal form due to Hatcher. In this paper, we determine the normal form in terms of crossings of partitions of ends corresponding to normal spheres, using a graph of trees representation for normal forms. In particular, we give a constructive proof of a criterion determining when a conjugacy class in $\pi_{2}(M)$ can be represented by an embedded sphere.
\end{abstract}

Keywords. Free groups; sphere complex; algebraic intersection numbers; graphs of trees.

\section{Introduction}

Topological and geometric methods have played a major role in the study of infinite groups. In particular, free (non-abelian) groups $\mathbb{F}_{n}$ of rank $n$ and the groups $\operatorname{Out}\left(\mathbb{F}_{n}\right)$ of their outer automorphisms have been extensively studied in analogy with fundamental groups of surfaces and the mapping class groups of surfaces. These groups have also been studied via a one-dimensional model which arises from regarding $\mathbb{F}_{n}$ as the fundamental group of a graph, for instance, see [1-4].

The 3-manifold $M=\sharp_{n} S^{2} \times S^{1}$ whose fundamental group is a free group of rank $n$, is used as a model for studying the free group and its automorphisms, in particular the group of outer automorphisms of free groups.

Embedded spheres in $M$ correspond to splittings of the free group. Our goal in this paper is to study the relation between such embedded spheres and partitions of the set of ends of the universal cover $\tilde{M}$ of $M$. Embedded spheres in $M$ can be represented in a normal form due to Hatcher. We show that such a sphere in normal form can be represented by a graph of trees, and show how the graph of trees is determined by crossings of partitions of the ends of $\tilde{M}$. As an application, we give a constructive proof of a criterion from [9] determining when a conjugacy class in $\pi_{2}(M)$ can be represented by an embedded sphere. In a sequel, we shall use the methods of this paper to construct the analogue of the space of geodesic laminations for surfaces and prove a compactness result for this space.

Our results here and in the sequel are motivated by the analogy between the group of outer automorphisms of the free group and the mapping class group of a surface, and consequently between the curve complex of a surface and the sphere complex of $M$. The curve complex of a surface $\Sigma$ is the simplicial complex with vertices, the isotopy classes of simple closed curves on $\Sigma$ and simplices corresponding to families of disjoint simple 
closed curves on $\Sigma$. Analogously, the sphere complex of $M$ is the simplicial complex whose vertices are the isotopy classes of embedded spheres in $M$, with isotopy classes of embedded spheres in $M$ deemed to span a simplex if they can be realized disjointly in $M$. The sphere complex is the splitting complex of the free group, with vertices corresponding to splittings and simplices to compatible splittings.

There have been two kinds of applications of the curve complex. On the one hand, the topological properties of the curve complex have been used to study the stable homology of the mapping class group in the work of Harer, Penner and others, [12], [13]. On the other hand, geometric properties of the curve complex have been used to study and construct various objects like mapping class groups, Teichmüller spaces and Thurston's compactification of Teichmüller space, for example [5], [26], [29], [30]. The geometric applications can be viewed in terms of the intersection number of curves on a surface.

For the case of the sphere complex, the analogues of the topological properties for the sphere complex have been proved and used by many, [14, 16-23]. Our goal is to develop the analogues of the geometric techniques used for the curve complex, based on the the algebraic intersection number of Scott and Swarup [31].

Specifically, in the sequel to this paper, we construct the analogue of the space of geodesic laminations and show that this space is compact. Many of the methods are parallel to those of this paper. In particular, we show in this paper that normal spheres correspond to finite subtrees of a specific tree together with some additional data. Further such a description is determined by data on crossing of spheres. Dropping the finiteness condition and modifying the notion of crossing leads to our generalization of spheres to geospheres, which is analogous to the passage from closed geodesics to all geodesics. Disjoint unions of such geospheres give laminations. We remark that an alternative approach based on the methods here, namely replacing graphs of trees by graphs of $\mathbb{R}$-trees, may also be fruitful.

We remark that a different geometric model, namely outer space, has been used extensively in the context of the free group (see, for instance, [6], [7]). In the case of surfaces, simple curves are both dimension one and co-dimension one objects, but this is not the case for free groups. Most of the geometric constructions for free groups in the literature are the 1-dimensional analogues of the construction for surfaces, but the constructions here are instead co-dimension 1 analogues.

We now outline the paper. The results in $\S \S 2-4$ are well-known in the field and are included for the sake of completeness. In $\S 2$, we see a description of $M$ and the notion of ends of $\tilde{M}$. In $\S 3$, we see that homology classes of embedded spheres in $\tilde{M}$ are completely determined by the partition of ends of $\tilde{M}$ associated to the spheres. In $\S 4$, we recall the notion of crossings of partitions of ends and make some simple observations.

In $\S 5$, given a partition $A$ of the set of ends of $\tilde{M}$, we construct a corresponding embedded sphere in $\tilde{M}$ which is in normal form in the sense of Hatcher, in terms of crossings of spheres, or equivalently of crossings of partitions of the set of ends of $\tilde{M}$. In $\S 6$, we recall some basic facts about the intersection number $c \cdot A$ between proper paths and homology classes and give a criterion for a class to be represented by an embedded sphere in $\tilde{M}$.

In §7, we show that a normal sphere can be described by a graph of trees, an analogue of the well-studied graph of groups. Finally, in $\S 8$, we give a constructive proof of the main result of [9], giving a criterion for a conjugacy class in $\pi_{2}(M)$ to be represented by an embedded sphere. The proof is constructive in the sense that a graph of trees structure is described in terms of crossing data for a conjugacy class in $\pi_{2}(M)$ satisfying the appropriate criteria, and is shown to correspond to a normal sphere representing the given class. 


\section{Preliminaries}

\subsection{The model 3-manifold}

Consider the 3-manifold $M=\sharp_{k} S^{2} \times S^{1}$, i.e., the connected sum of $k$ copies of $S^{2} \times S^{1}$. A description of $M$ can be given as follows: Consider the 3 -sphere $S^{3}$ and let $A_{i}, B_{i}, 1 \leq$ $i \leq k$, be a collection of $2 k$ disjoint embedded balls in $S^{3}$. Let $P$ be the complement of the union of the interiors of these balls and let $S_{i}$ (respectively, $T_{i}$ ) denote the boundary of $A_{i}$ (respectively, $B_{i}$ ). Then, $M$ is obtained from $P$ by gluing together $S_{i}$ and $T_{i}$ with an orientation reversing diffeomorphism $\varphi_{i}$ for each $i, 1 \leq i \leq k$. The image of $S_{i}$ (hence $T_{i}$ ) in $M$ will be denoted $\Sigma_{i}^{\prime}$. The fundamental group $\pi_{1}(M)$ of $M$, which is a free group of rank $k$, acts freely on the universal cover $\tilde{M}$ of $M$ by deck transformations.

\section{DEFINITION 2.1}

A smooth, embedded 2-sphere in $M$ is said to be essential if it does not bound a 3-ball in $M$.

\section{DEFINITION 2.2}

A system of 2-spheres in $M$ is defined to be a finite collection of disjointly embedded smooth essential 2-spheres $S_{i} \subset M$ such that no two spheres in this collection are isotopic.

Let $\Sigma=\cup_{j} \Sigma_{j}$ be a maximal system of 2-sphere in $M$. Let $M^{*}$ be obtained by splitting $M$ along $\Sigma$, i.e., $M^{*}$ is obtained from $M-\Sigma$ by completing with respect to the restriction of a Riemannian metric on $M$. Then, $M^{*}$ is a finite collection of 3-punctured 3-spheres $P_{k}$, whose boundary components correspond to the spheres $\Sigma_{i}$. Here, a 3-punctured 3-sphere is the complement of the interiors of three disjointly embedded 3-balls in a 3-sphere. Let $\tilde{M}^{*}$ be obtained similarly by splitting $\tilde{M}$ along standard spheres. Then $\tilde{M}^{*}$ is the disjoint union of lifts $\tilde{P}_{k}$ of the components $P_{k}$ of $M^{*}$.

There are natural inclusion maps $M^{*} \rightarrow M$ and $\tilde{M}^{*} \rightarrow \tilde{M}$. These maps are one-to-one on the union of the interiors of (the components of) $M^{*}$ and on each boundary component. Two boundary components are identified in pairs under these maps. Further, the map from $\tilde{M}^{*} \rightarrow M$ is injective on each component; we identify each component of $\tilde{M}^{*}$ with its image in $\tilde{M}$.

\subsection{Graphs and trees}

A graph can be described combinatorially as follows.

\section{DEFINITION 2.3}

A graph $\Gamma$ consists of two sets $E(\Gamma)$ and $V(\Gamma)$, called the edges and vertices of $\Gamma$, a mapping from $E(\Gamma)$ to $E(\Gamma)$ denoted $e \mapsto \bar{e}$, for which $e \neq \bar{e}$ and $\overline{\bar{e}}=e$, and a mapping from $E(\Gamma)$ to $V(\Gamma) \times V(\Gamma)$, denoted $e \mapsto(o(e), t(e))$, such that $\bar{e} \mapsto(t(e), o(e))$ for every $e \in E(\Gamma)$.

As is well-known we can associate to a graph a topological space. Geometrically, the set $E(\Gamma)$ is the set of oriented edges, with the pair of edges $e$ and $\bar{e}$ corresponding to a geometric edge (i.e., the homeomorphic image of an interval) with opposite orientations.

A tree is a graph so that the underlying topological space is contractible, or equivalently connected and simply connected. We shall use the following well-known characterization of trees. 


\section{PROPOSITION 2.4}

A graph $\Gamma$ is a tree if and only if it is connected and, for each edge e of $\Gamma$, the complement of the interior of e is not connected.

\subsection{Construction of the tree $T$}

We recall some constructions from [14].

We associate a tree $T$ to $\tilde{M}$ corresponding to the decomposition of $M$ by $\Sigma$. Let $\tilde{\Sigma}$ be the pre-image of $\Sigma$ in $\tilde{M}$. The vertices of the tree $T$ are of two types, with one vertex corresponding to each component of $\tilde{M}^{*}$ and one vertex for each component of $\tilde{\Sigma}$. An edge of $T$ joins a pair of vertices if one of the vertices corresponds to a component $X$ of $\tilde{M}^{*}$ and the other vertex corresponds to a component of $\tilde{\Sigma}$ that is in the image of the boundary of $X$. Thus, we have a $Y$-shaped subtree corresponding to each component of $\tilde{M}^{*}$. The end points of different $Y$ 's that correspond to the same sphere in $\tilde{\Sigma}$ are identified. We pick an embedding of $T$ in $\tilde{M}$ respecting the correspondences. The tree $T$ has bivalent and trivalent vertices with bivalent vertices corresponding to components of $\tilde{\Sigma}$.

\subsection{The space $K_{\tau}$}

Let $\tau$ be a finite subtree of $T$ such that all the terminal vertices of $\tau$ are bivalent in $T$. Let $K_{\tau}$ be the union of the images of components $\widetilde{P}_{k}$ which correspond to the vertices of $\tau$ which are trivalent in $T$. It is easy to see that $K_{\tau}$ is a compact,connected, simply-connected 3-manifold homeomorphic to a space of the form $S^{3}-\cup_{j=1}^{n} \operatorname{int}\left(D_{j}\right)$, with $D_{j}$ disjoint embedded balls in $S^{3}$. The closure $W_{i}$ of each complementary component of $K=K_{\tau}$ is non-compact. As $\tilde{M}$ is simply-connected and $K$ is connected, $N_{i}=\partial W_{i}$ is connected for each $W_{i}$. The boundary components $N_{i}$, and hence the complementary components $W_{i}$, correspond to the (finitely many) terminal vertices of $\tau$.

The space $\tilde{M}$ is the union of the spaces $K_{\tau}$, with $\tau$ a finite subtree of $T$. Henceforth, whenever we consider such a subtree $\tau$ of $T, K=K_{\tau}, W_{i}, N_{i}$ are as defined above.

\subsection{Ends of $\tilde{M}$}

We recall the notion of ends of a topological space. An end of a topological space is a point of the so called Freudenthal compactification of the space. It can be viewed as a way to approach infinity within the space.

Namely, let $X$ be a topological space. For a compact set $K \subset X$, let $C(K)$ denote the set of components of $X-K$. For $L$ compact with $K \subset L$, we have a natural map $C(L) \rightarrow C(K)$. Thus, as compact subsets of $X$ define a directed system under inclusion, we can define the set of ends $E(X)$ as the inverse limit of the sets $C(K)$. Further, we can compute the inverse limit with respect to any exhaustion by compact sets.

It is easy to see that a proper map $f: X \rightarrow Y$ induces a map $E(f): E(X) \rightarrow E(Y)$ and that if $f: X \rightarrow Y$ and $g: Y \rightarrow Z$ are proper maps, then $E(g \circ f)=E(g) \circ E(f)$. In particular, the real line $\mathbb{R}$ has two ends which can be regarded as $\infty$ and $-\infty$. Hence, a proper map $c: \mathbb{R} \rightarrow X$ gives a pair of ends $c_{-}$and $c_{+}$of $X$.

The space $\tilde{M}$ is non-compact and it has infinitely many ends. We denote the set of ends of $\tilde{M}$ by $E(\tilde{M})$. 


\subsection{Topology on the set of ends of $\tilde{M}$}

We define a topology on $E(\tilde{M})$. If $K$ is any compact subset of $\tilde{M}$, then $\tilde{M}-K$ has finitely many components and all the components are non-compact. Consider the collection of sets

$$
\mathcal{U}=\{E(W): W \text { a component of } M-K \text { for a compact set } K\} .
$$

It is easy to see that $\mathcal{U}$ forms a basis for a topology on $E(\tilde{M})$. The set $E(\tilde{M})$ is homeomorphic to a Cantor set, in particular, compact. Note that the set $E(T)$ of ends of $T$ can be identified with the set $E(\tilde{M})$.

\section{Embedded spheres in $\tilde{M}$ and partitions of ends of $\tilde{M}$}

Fix an orientation of $M$, and hence of $\tilde{M}$. We observe that, by the Hurewicz theorem [17], $\pi_{2}(M)=\pi_{2}(\tilde{M})=H_{2}(\tilde{M})$. So a class in $\pi_{2}(M)$ can be considered as a class in $\pi_{2}(\tilde{M})$ as well as a class in $H_{2}(\tilde{M})$. We shall implicitly use this identification throughout.

Given an oriented essential embedded sphere $S \subset \tilde{M}$, we get a partition of the set $E(\tilde{M})$ of $\tilde{M}$. Namely, as $H_{1}(\tilde{M})$ is trivial, it follows by Alexander duality that $\tilde{M}-S$ has two components, say $V^{+}$and $V^{-}$, with $V^{+}$on the positive side of $S$ according to the given orientations on $S$ and $\tilde{M}$. The sets $E^{ \pm}(S)$ are the sets of ends $E\left(V^{ \pm}\right)$of these components.

\section{PROPOSITION 3.1}

The sets $E^{ \pm}(S)$ are open in $E(\tilde{M})$.

Proof. As the sets $K_{\tau}$ form an exhaustion, there is a finite subtree $\tau$ of $T$ with all of its terminal vertices bivalent in $T$ such that $S$ is contained in $K=K_{\tau}$. Note that the set $E(\tilde{M})$ of ends of $\tilde{M}$ is partitioned into the sets $E\left(W_{i}\right)$, with $W_{i}$ the components of $\tilde{M}-K_{\tau}$. The sphere $S$ separates $K$ and gives a partition of the collection $\left\{N_{i}\right\}$ of boundary spheres of $K$. Note that interior of each $W_{i}$ is completely contained either in $V^{+}$or $V^{-}$and hence each set $E\left(W_{i}\right)$ lies entirely either inside $E^{+}$or inside $E^{-}$. Thus, both $E^{+}$and $E^{-}$are unions of basic open sets, hence are open.

As the sets $E^{ \pm}=E^{ \pm}(S)$ give a partition of $E(\tilde{M})$, both $E^{+}$and $E^{-}$are closed subsets of $E(\tilde{M})$. As $E(\tilde{M})$ is compact, both $E^{+}$and $E^{-}$are compact subsets of $E(\tilde{M})$.

Now, we shall see that the partition $\left\{E^{+}, E^{-}\right\}$of the set $E(\tilde{M})$ into open sets is completely determined by the homology class of $S$ in $\tilde{M}$. As $K$ is homeomorphic to the complement of the interiors of balls in $S^{3}$, by elementary algebraic topology the following holds.

Lemma 3.2. Orienting the boundary components of $K$ using induced orientations from $K$, the second homology group $\mathrm{H}_{2}(K)$ of $K$ is generated by the homology classes $\left[N_{i}\right]$ of the boundary spheres $N_{i}$ with the relation $\sum_{i}\left[N_{i}\right]=0$.

Let $S$ and $S^{\prime}$ be embedded spheres in $\tilde{M}$. There exists a finite subtree $\tau$ of $T$ with all of its terminal vertices bivalent in $T$, such that both $S$ and $S^{\prime}$ are contained in $K=K_{\tau}$. We shall require the following lemma:

Lemma 3.3. The homology classes of an oriented spheres in $K$ are in bijective correspondence with partitions of the collection of boundary spheres $N_{i}$. 
Proof. An embedded sphere $S$ separates $K$ into two components, $K_{+}$and $K_{-}$, with $K_{-}$ on the negative side of $S$. The homology class of $S$ can then be expressed as

$$
[S]=\sum_{i: N_{i} \subset \partial K_{-}}\left[N_{i}\right]
$$

Thus, the class is determined by the corresponding partition. Conversely, by Lemma 3.2, classes corresponding to different non-trivial partitions are different.

Now, we have the following proposition:

\section{PROPOSITION 3.4}

If $S^{\prime}$ is homologous to $S$ in $\tilde{M}$, then both $S$ and $S^{\prime}$ give the same partition of the set of ends of $\tilde{M}$.

Proof. If $S$ and $S^{\prime}$ are homologous in $\tilde{M}$, then as homology has compact support, we can choose a finite subtree $\tau$ of $T$ with all of its terminal vertices bivalent in $T$ such that they are homologous in $K=K_{\tau}$. So, by Lemma 3.3, they give the same partition of the collection of boundary spheres $N_{i}$ of $K$. Therefore, they give the same partition of the collection $\left\{E\left(W_{i}\right)\right\}$ and hence, give the same partition of the set $E(\tilde{M})$.

Conversely, we have the following.

\section{PROPOSITION 3.5}

If $S$ and $S^{\prime}$ give the same partition $\left(E^{+}, E^{-}\right)$of the set $E(\tilde{M})$ of ends of $\tilde{M}$, then $S$ and $S^{\prime}$ are homologous in $\tilde{M}$.

Proof. There exist a finite subtree $\tau$ of $T$, with all of its terminal vertices bivalent in $T$, such that both $S$ and $S^{\prime}$ are contained in $K=K_{\tau}$. The sphere $S$ separates $K$ and gives a partition of the boundary spheres $N_{i}$ of $K$ into two sets, which are determined by whether $E\left(W_{i}\right)$ lies (entirely) inside $E^{+}$or $E^{-}$. As the partition of the boundary components induced by $S^{\prime}$ is determined in the same fashion, $S$ and $S^{\prime}$ give the same partition of the boundary spheres of $K$. Hence, by Lemma 3.3, $S$ and $S^{\prime}$ are homologous in $K$ and therefore, homologous in $\tilde{M}$.

Now, we see that partitions of $E(\tilde{M})$ into two open sets correspond to embedded spheres in $\tilde{M}$. We require the following lemma.

Lemma 3.6. Given a partition of the set $E(\tilde{M})$ into two infinite closed (hence open) sets $E^{+}$and $E^{-}$, there exists a subtree $\tau \subset T$, with all the terminal vertices of $\tau$ bivalent in $T$, such that each $E\left(W_{i}\right)$ lies entirely either in $E^{+}$or $E^{-}$.

Proof. The sets $E^{+}$and $E^{-}$are both open and closed. As the set $E(\tilde{M})$ is compact, both $E^{+}$and $E^{-}$are compact. Let $e \in E^{+}$. As $E^{+}$is open, we can choose a finite tree $\tau_{e} \subset T$ with all the terminal vertices bivalent in $T$ such that $e$ is an element of the set $U_{e}$ of the ends of a component of $\tilde{M}-K_{\tau}$ and $U_{e} \subset E^{+}$. The sets $U_{e}$ for $e \in E^{+}$form an open cover of $E^{+}$. As $E^{+}$is compact, there exists finitely many basic open sets $U_{1}, \ldots, U_{n}$ which correspond to finite subtrees $\tau_{i}$, such that $E^{+}=\cup_{i=1}^{n} U_{i}$.

Let $\tau$ be a finite tree containing all the trees $\tau_{i}$, and let $K=K_{\tau}$ and $W_{i}$ be as before. We claim that $\tau$ is as required. For, if for some component $W_{i}, E\left(W_{i}\right)$ is not a subset of $E^{-}$, 
let $e \in E\left(W_{i}\right) \cap E^{+}$. Then $e \in U_{j} \subset E^{+}$for some $j, 1 \leq j \leq n$. As $\tau_{i} \subset \tau$ and $U_{j} \cap W_{i} \neq \phi\left(\right.$ as $e \in E\left(U_{j} \cap E\left(W_{j}\right)\right)$, it follows that $W_{i} \subset U_{j}$ (as $K_{\tau_{i}} \subset K_{\tau}$ ). This implies that $E\left(W_{i}\right) \subset E\left(U_{j}\right) \subset E^{+}$.

Thus, $E\left(W_{i}\right)$ must be a subset of either $E^{-}$or $E^{+}$, as required.

We now see the following:

\section{PROPOSITION 3.7}

Given a partition of the set $E(\tilde{M})$ into two infinite closed (hence open) sets $E^{+}$and $E^{-}$, there exist an embedded sphere $S$ in $\tilde{M}$, which gives this partition of $E(\tilde{M})$.

Proof. Suppose that $E^{+}$and $E^{-}$are two disjoint infinite closed subsets of $E(\tilde{M})$, which give a partition of $E(\tilde{M})$. By Lemma 3.6, we choose a subtree $\tau \subset T$ with all the terminal vertices of $\tau$ bivalent in $T$ such that each $E\left(W_{i}\right)$ lies entirely either in $E^{+}$or $E^{-}$. We can assign signs to $N_{i},+$ or - depending upon whether $E\left(W_{i}\right)$ lies inside $E^{+}$or $E^{-}$. Then, we can choose a sphere $S$ in $K$ which separates all positive signed $N_{i}$ from all negative signed $N_{i}$, as $K$ is homeomorphic to the complement in $S^{3}$ of the interior of finitely many 3-balls with boundary spheres $N_{i}$. It is easy to see that $S$ gives the partition of $E(\tilde{M})$ into the sets $E^{+}$and $E^{-}$.

\section{Crossings of spheres in $\tilde{M}$}

We next discuss the notion of crossings of embedded spheres in $\tilde{M}$ in terms of their associated partitions of ends of $\tilde{M}$.

Let $A$ and $B$ be two homology classes in $H_{2}(\tilde{M})$ represented by embedded spheres in $\tilde{M}$. We have seen that a homology class $A$ of an embedded sphere $S$ in $\tilde{M}$ is completely determined by a partition of $E(\tilde{M})$ into two open subsets of $E(\tilde{M})$. If $S$ gives partition of $E(\tilde{M})$ into two open subsets $E^{+}(S)$ and $E^{-}(S)$ of $E(\tilde{M})$, then we can write $E^{+}(A)=$ $E^{+}(S)$ and $E^{-}(A)=E^{-}(S)$.

\section{DEFINITION 4.1}

We say that $A$ and $B$ cross if, for each of the four pairs of signs $\varepsilon$ and $\eta$ in $\{+,-\}$, $E^{\varepsilon}(A) \cap E^{\eta}(B) \neq \phi$.

Suppose $A$ and $B$ do not cross, then for some choice of $\operatorname{sign} E^{\varepsilon}(A) \supset E^{\eta}(B)$. It follows that $E^{\bar{\varepsilon}}(A) \subset E^{\bar{\eta}}(B)$, where $\bar{\varepsilon}$ and $\bar{\eta}$ denote the opposite signs. Further, if $A \neq B$, then the inequalities are strict.

\section{DEFINITION 4.2}

We say that $B$ is on the positive side of $A$ if $E^{+}(A) \supset E^{\eta}(B)$ for some sign $\eta$. Otherwise, we say that $B$ is on the negative side of $A$. In general, we say that $B$ is on the $\varepsilon$-side of $A$ for the appropriate sign $\varepsilon$.

We shall need the following elementary observation.

Lemma 4.3. If $B$ is on the $\epsilon$-side of $A$ and $C$ is on the $\bar{\epsilon}$-side, then $B$ and $C$ do not cross.

Proof. For appropriate signs $\eta$ and $\xi, E^{\eta}(B) \subset E^{\epsilon}(A)$ and $E^{\xi}(C) \subset E^{\bar{\epsilon}}(A)$. Hence, $E^{\eta}(B) \cap E^{\xi}(C)=\phi$, which shows that $B$ and $C$ do not cross. 
If $B$ is on the $\epsilon$-side of $A$ and $C$ is on the $\bar{\epsilon}$-side, then we say that $A$ is between $B$ and $C$.

\section{PROPOSITION 4.4}

Let $A$ and $B$ be two homology classes in $H_{2}(\tilde{M})$ represented by embedded spheres in $\tilde{M}$. Then, $A$ and $B$ are represented by disjoint embedded spheres in $\tilde{M}$ if and only if $A$ and $B$ do not cross.

Proof. Suppose $A$ and $B$ are represented by embedded spheres $S$ and $S^{\prime}$ respectively. Denote the closures of the components of the complement of $S$ (respectively, $S^{\prime}$ ) by $X_{1}$ and $X_{2}$ (respectively, $Y_{1}$ and $\left.Y_{2}\right)$ so that $E\left(X_{1}\right)=E^{+}(A)=E^{+}(S)$ and $E\left(X_{2}\right)=E^{-}(A)=$ $E^{-}(S)$, and similarly $E\left(Y_{1}\right)=E^{+}(B)=E^{+}\left(S^{\prime}\right)$ and $E\left(Y_{2}\right)=E^{-}(B)=E^{-}\left(S^{\prime}\right)$.

Suppose $S$ and $S^{\prime}$ are disjoint embedded spheres in $\tilde{M}$. Without loss of generality, suppose $S^{\prime}$ is contained in the interior of $X_{1}$. Then, the component $Y_{i}$ which does not intersect $S$ is completely contained inside $X_{1}$. Without loss of generality, let this component be $Y_{1}$. Then, $E^{+}(B)=E\left(Y_{1}\right) \subseteq E\left(X_{1}\right)=E^{+}(A)$. This implies $E^{+}(B) \cap E^{-}(A)=\phi$. Similarly, we can see in all other cases at least one of the four sets $E^{\varepsilon}(A) \cap E^{\eta}(B)$, for $\varepsilon$ and $\eta$ obtained by choosing signs $\varepsilon$ and $\eta$ in $\{+,-\}$, is empty.

Conversely, suppose $A$ and $B$ do not cross. We shall show that $A$ and $B$ can be represented by disjoint embedded spheres in $\tilde{M}$. Let $\tau$ be a finite subtree of $T$ with all of its terminal vertices bivalent in $T$, such that both $A$ and $B$ are supported in $K=K_{\tau}$. Each set $E\left(W_{i}\right)$ is completely contained either in $E^{+}(A)$ or $E^{-}(A)$. Similarly, each set $E\left(W_{i}\right)$ is completely contained either in $E^{+}(B)$ or $E^{-}(B)$. We can assign signs $+_{A}$ or $-{ }_{A}$ to each component $N_{i}$ of $\partial K$ depending on whether $E\left(W_{i}\right)$ lies inside $E^{+}(A)$ or $E^{-}(A)$. Similarly, we can assign signs $+_{B}$ or $-_{B}$ to $N_{i}$, depending upon whether $E\left(W_{i}\right)$ lies inside $E^{+}(B)$ or $E^{-}(B)$. Thus, the collection of boundary spheres $N_{i}$ of $K$ get partitioned into two sets $U_{A}^{+}$ and $U_{A}^{-}$containing $+_{A}$ signed and $-_{A}$ signed boundary spheres, respectively. Similarly, the collection of boundary spheres $N_{i}$ of $K$ get partitioned into two sets $U_{B}^{+}$and $U_{B}^{-}$ containing $+_{B}$ signed and $-_{B}$ signed boundary spheres, respectively. As $A$ and $B$ do not cross, for some choice of sign, $E^{\varepsilon}(A) \supset E^{\eta}(B)$. Suppose $E^{+}(A) \subset E^{+}(B)$. This implies $U_{A}^{+} \subset U_{B}^{+}$. It also follows that $U_{B}^{-} \subset U_{A}^{-}$.

Let $S$ be a sphere separating $U_{A}^{+}$from $U_{A}^{-}$. Then a complementary component $V$ of $S$ has boundary components $S$ and the boundary spheres in $U_{A}^{-}$. We take $S^{\prime}$ to be the sphere in $V$ separating $U_{B}^{-} \subset U_{A}^{-}$from $\left(U_{B}^{+}-U_{A}^{+}\right) \cup S$. Then $S^{\prime}$ is disjoint from $S$ and induces the partition corresponding to $B$.

Thus, we get two disjointly embedded spheres $S$ and $S^{\prime}$ representing the homology classes $A$ and $B$. The other cases are similar.

\section{Partition of ends and normal forms}

This section is devoted to associating to a partition $A=\left(E^{+}(A), E^{-}(A)\right)$ of the space $E(\tilde{M})$ of ends of $\tilde{M}$ into non-empty open sets, an embedded sphere $S$ in $\tilde{M}$ which is in normal form in the sense of Hatcher, so that $E^{ \pm}(S)=E^{ \pm}(A)$. Along the way, we see what data determines a sphere in normal form in $\tilde{M}$ and the relation between this data, partitions of ends and crossings. Specifically, we prove the following:

Theorem 5.1. Given a partition $A=\left(E^{+}(A), E^{-}(A)\right)$ of the set $E(\tilde{M})$ of ends of $\tilde{M}$ into two non-empty open sets, there is a normal sphere $S$ in $\tilde{M}$ so that $A$ is the partition given by the ends of the components of $\tilde{M}-S$. 
This result can be seen by taking an embedded sphere corresponding to the partition and making this in normal form using Hatcher's results. Our purpose in this section is to show how one can directly construct the sphere using the data of crossings.

We have seen that embedded spheres correspond to partitions of ends. So, we can talk about crossing of a partition $A$ of $E(\tilde{M})$ and sphere $S$. We now turn to the construction of the normal sphere representing the partition $A$.

We recall the notion of normal sphere systems from [14]. Let $\Sigma=\cup_{j} \Sigma_{j}$ be a maximal system of 2 -spheres in $M$. We recall that the manifold $M^{*}$ obtained by splitting $M$ along $\Sigma$ is a finite union of disjoint 3-punctured 3-spheres $P_{k}$, whose interiors $\operatorname{int}\left(P_{k}\right)$ are embedded in $M$. Suppose $S$ is a surface in $M$ which is transversal to the spheres $\Sigma_{i}$. Then the closure $F_{k}$ in $P_{k}$ of $\operatorname{int}\left(P_{k}\right) \cap S$ is a properly embedded, not necessarily connected surface. We call the components of $F_{k}$ the pieces of $S$ in $P_{k}$.

\section{DEFINITION 5.2}

A system of 2-spheres $S=\cup_{i} S_{i}$ in $M$ is said to be in normal form with respect to $\Sigma$ if each $S_{i}$ either coincides with a sphere $\Sigma_{j}$ or meets $\Sigma$ transversely such that the following two conditions hold in each $P_{k}$ :

(1) Each piece in $P_{k}$ meets each component of $\partial P_{k}$ in at most one circle.

(2) No piece in $P_{k}$ is a disk which is isotopic, fixing its boundary, to a disk in $\partial P_{k}$.

Thus, each piece is a disk, a cylinder or a pair of pants. A disk piece has its boundary on one component of $\partial P_{k}$ and separates the other two components of $\partial P_{K}$. A cylinder piece joins two boundary components of $\partial P_{k}$ and a pants piece joins all three boundary spheres of $P_{k}$.

Similarly, we can define sphere systems in normal form with respect to the pre-image $\tilde{\Sigma}$ of $\Sigma$ in $\tilde{M}$. We call the spheres $\Sigma_{i}$ standard spheres in $M$ and their lifts $\widetilde{\Sigma}_{i}$ standard spheres in $\tilde{M}$.

Recall the following result from [14].

\section{PROPOSITION 5.3 (Hatcher)}

Every system $S \subset M$ can be isotoped to be in normal form with respect to $\Sigma$. In particular, every embedded sphere $S$ which does not bound a ball in $M$ can be isotoped to be in normal form with respect to $\Sigma$.

Given a sphere $S$ in normal form with respect to $\Sigma$ in $M$ and a lift $\tilde{S}$ of $S$ to $\tilde{M}$, we associate a tree $T(\tilde{S})$ corresponding to the decomposition of $\tilde{S}$ into pieces. The tree $T(\tilde{S})$ has two kinds of vertices, one for each piece $P \subset \widetilde{P}_{k}$ and one for each component $C$ of $\tilde{S} \cap \widetilde{\Sigma_{i}}$ for standard spheres $\tilde{\Sigma}_{i}$. Edges join vertices corresponding to components $P$ to those corresponding to $C \subset \partial P$. Here we identify $P$ with its image in $\tilde{M}$.

In [14], it is shown that $T(\tilde{S})$ is a tree. Moreover, the inclusion $\tilde{S} \hookrightarrow \tilde{M}$ induces a natural inclusion map $T(\tilde{S}) \hookrightarrow T$, in particular each $\widetilde{P}_{k}$ contains at the most one piece of $\tilde{S}$. So, we can view $T(\tilde{S})$ as a subtree of $T$. It is easy to see that, as with Hatcher's result mentioned above, if $S^{\prime}$ is a normal sphere in $\tilde{M}$, then the map $T\left(S^{\prime}\right) \rightarrow T$ is injective and, in particular, each $\widetilde{P}_{k}$ contains at most one piece of $S^{\prime}$. If $S$ is a standard sphere (or can be isotoped to the standard sphere), then the associated tree $T(\tilde{S})$ is the single vertex in $T$ corresponding to that standard vertex.

Our construction is motivated by the following lemma from [10]. 
Lemma 5.4. Let $S$ be a normal sphere in $\tilde{M}$ and let $\tilde{\Sigma}_{i}$ be a standard sphere.

(1) The spheres $S$ and $\tilde{\Sigma}_{i}$ intersect if and only if they cross.

(2) If $S$ and $\tilde{\Sigma}_{i}$ intersect, they intersect transversely in a circle $S^{1}$.

Thus, if $A$ is represented by a normal sphere $S$ in $\tilde{M}$, we can determine the intersection of $S$ with each standard sphere in $\tilde{M}$, in terms of crossings. The standard spheres in $\tilde{M}$ correspond to the bivalent vertices of the tree $T$, which we call standard vertices. Now, we associate a subgraph $\tau$ of $T$ to $A$ as follows: If $A$ crosses the standard sphere $\widetilde{\Sigma_{i}}$, then $\tau$ contains the bivalent vertex $v_{i}$ corresponding to $\widetilde{\Sigma_{i}}$ and the edges $e_{1}^{i}$ and $e_{2}^{i}$ containing that vertex $v_{i}$. The other vertex $v_{j}^{i}$ of each edge $e_{j}^{i}, j=1,2$ is a trivalent vertex in $T$ which corresponds to a component of $\tilde{M}^{*}$. Each $v_{j}^{i}$ may be univalent (terminal), bivalent or trivalent in $\tau$. Note that each terminal vertex of $\tau$ is trivalent in $T$.

If $A$ does not cross some standard sphere in $\tilde{M}$, then $\tau$ does not contain the standard vertex corresponding to this standard sphere and hence does not contain the edges containing this standard vertex. Note that any edge $e$ in $T$ contains a unique standard (bivalent) vertex of $T$.

We first consider the degenerate case where $A$ does not cross any standard sphere.

Lemma 5.5. Suppose $A=\left(E^{+}, E^{-}\right)$is a partition of $E(\tilde{M})$ into two open sets such that $E^{+} \neq \phi \neq E^{-}$. Suppose no standard sphere crosses $A$. Then, there exists a standard sphere $\Sigma_{0}$ such that $E^{ \pm}=E^{ \pm}\left(\Sigma_{0}\right)$.

Proof. By hypothesis, if $v$ is a standard bivalent vertex of $T$, the standard sphere $\Sigma(v)$ corresponding to $v$ does not cross $A$. Hence, after choosing orientations appropriately, either $E^{+}(\Sigma(v)) \subset E^{+}$or $E^{-}(\Sigma(v)) \subset E^{-}$. If $\Sigma(v)=\Sigma_{0}$ satisfies both the conditions, then $E^{ \pm}=E^{ \pm}\left(\Sigma_{0}\right)$.

Suppose no $\Sigma(v)$ satisfies both the above conditions, we get a partition of bivalent vertices of $T$ as

$$
V^{+}=\left\{v: E^{+}(\Sigma(v)) \subset E^{+}\right\}
$$

and

$$
V^{-}=\left\{v: E^{-}(\Sigma(v)) \subset E^{-}\right\} .
$$

Let $X^{ \pm}$be the union of all the edges $e$ in $T$ such that the bivalent vertex of $e$ lies in $V^{ \pm}$. Then the sets $X^{ \pm}$are closed and $T=X^{+} \cup X^{-}$. Hence, $X^{+} \cap X^{-} \neq \phi$. By construction, $X^{+} \cap X^{-}$consists of trivalent vertices of $T$. Let $w \in X^{+} \cap X^{-}$and let $v_{1}, v_{2}$ and $v_{3}$ be bivalent vertices adjacent to $w$. Note that at least one $v_{i} \in V^{+}$and at least one $v_{j} \in V^{-}$. Without loss of generality, suppose $v_{1}, v_{2} \in V^{+}$and $v_{3} \in V^{-}$.

Let $N(w)$ denote the set of all the points in $T$ with distance at most 1 from $w$. Then, $T-N(w)$ has three components $V_{1}, V_{2}$ and $V_{3}$ whose closures contain the vertices $v_{1}, v_{2}$ and $v_{3}$, respectively. It is easy to see that $E\left(V_{1}\right) \subset E^{+}, E\left(V_{2}\right) \subset E^{+}$and $E\left(V_{3}\right) \subset E^{-}$. It follows that $E^{+}\left(\Sigma\left(v_{3}\right)\right)=E^{+}\left(\Sigma\left(v_{1}\right)\right) \cup E^{+}\left(\Sigma\left(v_{2}\right)\right)$. This implies $E^{+}\left(\Sigma\left(v_{3}\right)\right) \subset E^{+}$. As $v_{3} \in V^{-}, E^{-}\left(\Sigma\left(v_{3}\right)\right) \subset E^{-}$. But then, $v_{3} \in V^{+} \cap V^{-}$. This is a contradiction as $V^{+}$ and $V^{-}$are disjoint. Hence, there must exist a standard sphere $\Sigma_{0}$ such that $E^{ \pm}=E^{ \pm}\left(\Sigma_{0}\right)$.

Thus, if $A$ does not cross any standard sphere, the tree $\tau$ associated to $A$ is a standard vertex corresponding to the standard sphere representing $A$. 
A normal sphere $S$ in $\tilde{M}$ has connected intersection with each set $\widetilde{P}_{k}$. The piece of $S$ in $\widetilde{P}_{k}$ is a disc $D$, an annulus (which we call a tube) $A$ or a thrice punctured 2-sphere $Y$ (which we call a $Y$-piece or pant piece) according as the number of edges in $\tau(S)$ adjoining $v$ corresponding to $\widetilde{P}_{k}$ is 1,2 or 3 . We first make some observations about these cases.

Firstly, suppose $v$ is a vertex of $\tau$ adjacent to a single edge $e_{0} \in \tau$, i.e., $v$ is a terminal vertex of $\tau$ (hence trivalent in $T$ ). Let $v_{0} \in \tau$ be the other vertex of $e_{0}$ and $\Sigma_{0}$ be the standard sphere in $\tilde{M}$ corresponding to $v_{0}$. Then, $A$ crosses $\Sigma_{0}$. Let the other edges adjacent to $v$ in $T$ be $e_{1}$ and $e_{2}$ with bivalent vertices $v_{1}$ and $v_{2}$, respectively. Consider the standard spheres $\tilde{\Sigma}_{i}=\tilde{\Sigma}\left(v_{i}\right)$ corresponding to the vertices $v_{i}$, with orientations chosen so that for $i=1,2$, the set $E^{+}\left(\tilde{\Sigma}_{i}\right)$ is the set of ends of the component of $\tilde{M}-\tilde{\Sigma}_{i}$ that does not contain $\tilde{\Sigma}_{0}$. We can orient $\tilde{\Sigma}_{0}$ so that $E^{+}\left(\tilde{\Sigma}_{0}\right)=E^{+}\left(\tilde{\Sigma}_{1}\right) \cup E^{+}\left(\tilde{\Sigma}_{2}\right)$.

Lemma 5.6. For some sign $\epsilon, E^{\epsilon}(A) \supset E^{+}\left(\tilde{\Sigma}_{1}\right)$ and $E^{\bar{\epsilon}}(A) \supset E^{+}\left(\tilde{\Sigma}_{2}\right)$.

Proof. First note that for any choice of sign $\eta \in\{-,+\}, E^{+}\left(\tilde{\Sigma}_{i}\right) \not \supset E^{\eta}(A)$, for $i=$ 1,2 , as if $E^{+}\left(\tilde{\Sigma}_{i}\right) \supset E^{\eta}(A)$, then $E^{+}\left(\tilde{\Sigma}_{0}\right) \supset E^{\eta}(A)$, contradicting the hypothesis that $A$ crosses $\left[\tilde{\Sigma}_{0}\right]$. Hence, as $A$ does not cross the spheres $\tilde{\Sigma}_{i}$, for appropriate signs $\epsilon_{i}$, $E^{\epsilon_{i}}(A) \supset E^{+}\left(\tilde{\Sigma}_{i}\right)$ for $i=1$, 2. Finally, if $\epsilon_{1}=\epsilon_{2}=\epsilon$, then $E^{\epsilon}(A) \supset E^{+}\left(\tilde{\Sigma}_{0}\right)$ as $E^{+}\left(\tilde{\Sigma}_{0}\right)=E^{+}\left(\tilde{\Sigma}_{1}\right) \cup E^{+}\left(\tilde{\Sigma}_{2}\right)$, contradicting the hypothesis that $A$ crosses $\tilde{\Sigma}_{0}$.

Thus, one of the spheres $\tilde{\Sigma}_{1}$ and $\tilde{\Sigma}_{2}$ is on the positive side of $A$ and the other on the negative side.

Consider next the case of a vertex $v$ of valence 2 of $\tau$ which is trivalent in $T$. Then there is an edge $e_{v}$ of $T$ adjacent to $v$ which is not in $\tau$. The standard sphere $\tilde{\Sigma}\left(e_{v}\right)$ corresponding to the other end vertex of the edge $e_{v}$ is either on the positive side of $A$ or on the negative side (as it does not cross $A$ ).

We shall see that $\tau$ is a tree and the partition $A$ is determined by $\tau$ together with data of the above form at terminal and non-standard bivalent vertices of $\tau$. The standard bivalent vertices of $\tau$ are standard (bivalent) vertices of $T$. We begin by showing that $\tau$ is a tree. As this is a subgraph of a tree $T$, it suffices to show that $\tau$ is connected.

Lemma 5.7. The subgraph $\tau \subset T$ is connected, hence a tree.

Proof. Suppose $\tau$ is not connected. As $\tau$ is a subgraph of $T$, there is a standard vertex $v \notin \tau$ such that both components $X_{1}$ and $X_{2}$ of $T-v$ intersect $\tau$. Let $\tau_{i}=\tau \cap X_{i}$, for $i=1,2$. Let $\tilde{\Sigma}_{0}=\tilde{\Sigma}(v)$.

As $v \notin \tau, A$ does not cross $\tilde{\Sigma}_{0}$. Hence, we can orient $\tilde{\Sigma}_{0}$ so that for some sign $\epsilon$, $E^{+}\left(\tilde{\Sigma}_{0}\right) \subset E^{\epsilon}(A)$. Without loss of generality, $E^{+}\left(\tilde{\Sigma}_{0}\right)$ is the set of ends of $X_{1}$.

Let $\tau^{\prime}$ be the convex hull of the vertex $v$ and $\tau_{1}$. As $\tau$ is a finite graph, $\tau^{\prime}$ is a finite tree. Let $w$ be a terminal vertex of $\tau^{\prime}$ distinct from $v$. Then, $w$ is a terminal vertex of $\tau$. Hence, by Lemma 5.6, there is an adjacent edge $e^{\prime} \notin \tau$ with its other end vertex $v^{\prime}$ such that $E^{+}\left(\tilde{\Sigma}\left(v^{\prime}\right)\right) \subset E^{\bar{\epsilon}}(A)$, with the orientation chosen so that $E^{+}\left(\tilde{\Sigma}\left(v^{\prime}\right)\right)$ is the set of ends of the component of $\tilde{M}-\tilde{\Sigma}\left(v^{\prime}\right)$ that does not intersect $\tau$. It follows that $E^{+}\left(\tilde{\Sigma}\left(v^{\prime}\right)\right) \subset E^{+}\left(\tilde{\Sigma}_{0}\right)$, and hence, $E^{+}\left(\tilde{\Sigma}_{0}\right) \cap E^{\bar{\epsilon}}(A) \neq \phi$, a contradiction.

We next see that $\tau$ is a finite tree.

Lemma 5.8. The tree $\tau$ is compact, hence finite.

Proof. If $\tau$ is not compact, then some end $P \in E(\tilde{M})=E(T)$ is an end of $\tau$. Without loss of generality $P \in E^{+}(A)$. As $E^{+}(A)$ is open in the space of ends of $T$, there is a finite 
connected tree $\kappa \subset T$ and a component $V$ of $T-\kappa$ so that $P \in E(V) \subset E^{+}(A)$. We shall show that no edge of $V$ is contained in $\tau$, contradicting the assumption that $P$ is an end of $\tau$.

Let $e$ be an edge of $T$ contained in $V=T-\kappa$. Then, as $\kappa$ is connected, some component $W$ of $T-e$ is disjoint from $\kappa$, and hence contained in $V$. Suppose $v$ is the vertex of $e$ such that $v$ is a standard bivalent vertex in $T$. If $\Sigma(v)$ is the standard sphere corresponding to $v$, then it follows that for some sign $\varepsilon, E^{\varepsilon}(\Sigma(v)) \subset E(V) \subset E^{+}(A)$, and hence, $\Sigma(v)$ does not cross $A$. This implies $v$ is not in $\tau$. It follows that $e$ is not in $\tau$. Thus, no edge of $V$ is in $\tau$, as required.

Thus, we have a finite tree $\tau$ associated to the partition $A$, with the terminal vertices of $\tau$ trivalent in $T$. We shall next show that the partition $A$ is determined by the tree together with additional data for vertices adjoining the tree.

Let $U(\tau)$ and $B(\tau)$ denote the univalent (terminal) and non-standard bivalent vertices of $\tau$, respectively. Let $N(\tau)$ be the subgraph of $T$ consisting of points with distance at most 1 from $\tau$. Then, $N(\tau)$ is a tree, which is the union of $\tau$ with the following two kinds of edges:

(1) For each univalent vertex $v \in U(\tau)$ of $\tau$, we have a pair of edges $e_{1}(v) \notin \tau$ and $e_{2}(v) \notin \tau$ with $v$ as a vertex. Let $v_{1}$ and $v_{2}$ be the other vertices of $e_{1}$ and $e_{2}$, respectively.

(2) For each non-standard bivalent vertex $w \in B(\tau)$ of $\tau$, we have an edge $e(w) \notin \tau$ with $w$ as an end-vertex. Let $w_{1}$ be its other end vertex.

By Lemma 5.6, for a terminal vertex $v$, the sphere corresponding to exactly one of $v_{1}$ and $v_{2}$ is on the positive side of $\tau$ (positive side of $A$ ). The vertices $v_{1}$ and $v_{2}$ are end vertices of $e_{1}$ and $e_{2}$ respectively. So, we can assign positive or negative signs to these edges accordingly. We denote the positive edge by $e_{+}(v)$ and denote the other edge (which is on the negative side) by $e_{-}(v)$. We denote the standard spheres corresponding to $v_{1}$ and $v_{2}$ by $\tilde{\Sigma}\left(v_{1}\right)=\tilde{\Sigma}\left(e_{1}\right)$ and $\tilde{\Sigma}\left(v_{2}\right)=\tilde{\Sigma}\left(e_{2}\right)$, respectively. For a non-standard bivalent vertex $w$ of $\tau$, we can associate a sign $\epsilon(w)$ so that $\tilde{\Sigma}\left(w_{1}\right)=\tilde{\Sigma}(e(w))$ is on the $\epsilon(w)$-side of $A$.

We show that the tree $\tau$ together with the additional data determines a partition of the ends, which coincides with the given partition.

Lemma 5.9. The partition $A$ is determined by $\tau$ together with the functions $e_{+}(v), v \in$ $U(\tau)$ and $\epsilon(w), w \in B(\tau)$.

Proof. The spheres $\tilde{\Sigma}\left(e_{ \pm}(v)\right)$ for terminal vertices of $\tau$ together with $\tilde{\Sigma}(e(w))$ for nonstandard bivalent vertices of $\tau$ separate $\tilde{M}$ into a compact component corresponding to $\tau$ and one non-compact component for each such sphere $\tilde{\Sigma}$. We can orient the spheres $\tilde{\Sigma}$ so that the compact component is on the negative side of $\tilde{\Sigma}$. Then, the set of ends of the non-compact component is $E^{+}(\tilde{\Sigma})$. Hence, we have a partition

$E(\tilde{M})=\left[\bigcup_{v \in B(\tau)}\left(E^{+}\left(\tilde{\Sigma}\left(e_{+}(v)\right)\right) \cup E^{+}\left(\tilde{\Sigma}\left(e_{-}(v)\right)\right)\right)\right] \cup\left[\bigcup_{w \in U(\tau)} E^{+}(\tilde{\Sigma}(e(w)))\right]$.

By construction, $E^{+}\left(\tilde{\Sigma}\left(e_{+}(v)\right)\right) \subset E^{+}(A)$ and $E^{+}\left(\tilde{\Sigma}\left(e_{-}(v)\right)\right) \cap E^{+}(A)=\phi$, for each terminal vertex $v$ of $\tau$. For each non-standard bivalent vertex $w$ of $\tau, E^{+}(\tilde{\Sigma}(e(w))) \subset$ $E^{+}(A)$ if $\epsilon(w)=+$ and $E^{-}(\tilde{\Sigma}(e(w))) \cap E^{+}(A)=\phi$ otherwise. Hence,

$$
E^{+}(A)=\bigcup_{v \in B(\tau)} E^{+}\left(\tilde{\Sigma}\left(e_{+}(v)\right)\right) \cup \bigcup_{\{w \in U(\tau): \epsilon(w)=+\}} E^{+}(\tilde{\Sigma}(e(w))) .
$$

This is determined by the given data. Hence, the partition $A$ is determined by the given data. 
It is now easy to construct a normal sphere $S$ in $\tilde{M}$ representing the partition $A$. Note that a normal sphere $S$ represents the partition $A$ if and only if $E^{ \pm}(S)=E^{ \pm}(A)$.

We can associate to an oriented normal sphere $S \subset \tilde{M}$ data very similar to that associated to the partition $A$. Firstly, the sphere $S$ has support which is a subtree $\tau$. A terminal vertex $v$ of $\tau$ corresponds to a disc piece in a thrice-punctured 3-sphere $P(v)$, which separates the two other boundary components of $P(v)$. Exactly one of these lies on the positive side. Thus, as the boundary components correspond to vertices of $T$ adjacent to $v$, we get a pair of edges $e_{ \pm}(v)$. A non-standard bivalent vertex $w$ of $\tau$ corresponds to an annulus piece (cylinder piece) in $P(w)$. The boundary component of $P(w)$ not intersecting the annulus is on either the positive or the negative side of $S$, giving a sign $\epsilon(w)$. A trivalent vertex $w^{\prime}$ corresponds to a pants piece in the 3-punctured 3-sphere $P\left(w^{\prime}\right)$.

Lemma 5.10. Given a finite tree $\tau$, associated data $e_{+}(v)$ and $\epsilon(w)$, and an oriented circle on the sphere $\tilde{\Sigma}\left(V^{\prime}\right)$ for each non-terminal vertex $v^{\prime}$ of $\tau$, there is an oriented normal sphere $S \subset \tilde{M}$ with corresponding data $\tau, e_{+}$and $\epsilon$ and whose restriction to each thricepunctured 3-sphere has boundary the corresponding oriented circles. Further, the partition corresponding to $S$ is the one corresponding to the data $\tau, e_{+}$and $\epsilon$.

Proof. To each standard bivalent vertex of the tree $\tau$, we associate a circle in the corresponding standard sphere which we co-orient according to the given partition. For each trivalent vertex $v$ of $\tau$, we associate a pants piece with boundary the circles in the standard sphere that have been constructed.

Next, if $v$ is a non-standard bivalent vertex of $\tau$, the two adjacent standard vertices correspond to circles on two standard spheres. We join them by an annulus so that the other standard sphere bounding the 3-holed sphere corresponding to $v$ is on the side of the annulus given by $\epsilon(v)$. Similarly, for a terminal vertex of $\tau$ we consider a disc so that the standard spheres corresponding to adjacent vertices of $T$ that are not in $\tau$ is on the positive side of the disc and the one corresponding to the other adjacent vertex is on the negative side.

\section{Embedding classes, intersection number of a proper path and homology classes}

In this section, we recall and sketch a proof of a theorem of [9] characterizing when a conjugacy class in $\pi_{2}(M)$ can be represented by an embedded sphere in $\tilde{M}$.

\subsection{Intersection number of a proper path and homology classes}

Consider a proper map $c: \mathbb{R} \rightarrow \tilde{M}$. As $\tilde{M}$ is a union of the simply-connected compact sets $K_{\tau}$, where $\tau$ is a finite subtree of $T$, the following lemma is straightforward.

Lemma 6.1. There is a one-to-one correspondence between proper homotopy classes of maps $c: \mathbb{R} \rightarrow \tilde{M}$ and pairs $\left(c_{-}, c_{+}\right) \in E(\tilde{M}) \times E(\tilde{M})$.

Let $A \in H_{2}(\tilde{M})=\pi_{2}(\tilde{M})$. Represent $A$ by a (not necessarily connected) surface in $\tilde{M}$ (also denoted $A$ ). Given a proper map $c: \mathbb{R} \rightarrow \tilde{M}$ which is transversal to $A$. We consider the algebraic intersection number $c \cdot A$. This depends only on the homology class of $A$ and the proper homotopy class of $c$. The proper map $c: \mathbb{R} \rightarrow \tilde{M}$ gives a pair of ends $c_{-}$and $c_{+}$of $\tilde{M}$. We shall refer to $c$ as a proper path from $c_{-}$to $c_{+}$or as a proper path 
joining $c_{-}$and $c_{+}$. We denote such a path $c$ by $\left(c_{-}, c_{+}\right)$. The path $\left(c_{-}, c_{+}\right)$is unique up to proper homotopy. In particular, for a homology class $A \in H_{2}(\tilde{M})$, the intersection number $\left(c_{-}, c_{+}\right) \cdot A$ is well-defined and can be computed using any proper path joining $c_{-}$and $c_{+}$. For details, see [9].

As $H_{1}(\tilde{M})=0$, a homology class $A$ is determined by the intersection numbers $c \cdot A$, where $c: \mathbb{R} \rightarrow \tilde{M}$ is a proper path. The following result of [9] characterizes which classes in $H_{2}(\tilde{M})$ can be represented by embedded spheres in $\tilde{M}$.

Theorem 6.2. The class $A \in H_{2}(\tilde{M})$ can be represented by an embedded sphere in $\tilde{M}$ if and only if, for each proper map $c: \mathbb{R} \rightarrow \tilde{M}, c \cdot A \in\{0,1,-1\}$.

We have seen that an embedded sphere $S$ in $\tilde{M}$, partitions $\tilde{M}$ into two components with closure of each component non-compact, giving a partition of the ends of $\tilde{M}$ into $E^{ \pm}(S)$. It is easy to see that if $c: \mathbb{R} \rightarrow \tilde{M}$ is a proper path, then

- if $c_{-} \in E^{-}(S)$ and $c_{+} \in E^{+}(S)$, then $c \cdot S=1$,

- if $c_{-} \in E^{+}(S)$ and $c_{+} \in E^{-}(S)$, then $c \cdot S=-1$,

- if $c_{-} \in E^{-}(S)$ and $c_{+} \in E^{-}(S)$, then $c \cdot S=0$,

- if $c_{-} \in E^{+}(S)$ and $c_{+} \in E^{+}(S)$, then $c \cdot S=0$.

In particular, $c \cdot S \in\{0,1,-1\}$.

Conversely, let $A$ be a homology class satisfying the hypothesis of the theorem. We shall construct a normal sphere in $\tilde{M}$ that represents $A$.

The first step is the following elementary lemma, whose proof is in [9].

Lemma 6.3. There is a partition $E^{ \pm}(A)$ of the set $E(\tilde{M})$ of ends $\tilde{M}$ so that

- if $c_{-} \in E^{-}(A)$ and $c_{+} \in E^{+}(A)$, then $c \cdot A=1$,

- if $c_{-} \in E^{+}(A)$ and $c_{+} \in E^{-}(A)$, then $c \cdot A=-1$,

- if $c_{-} \in E^{-}(A)$ and $c_{+} \in E^{-}(A)$, then $c \cdot A=0$,

- if $c_{-} \in E^{+}(A)$ and $c_{+} \in E^{+}(A)$, then $c \cdot A=0$.

Thus, we have a partition of the ends just as in the case of embedded spheres. By Theorem 5.1, this corresponds to the partition given by a normal sphere $S$ in $\tilde{M}$. As homology classes are determined by their associated partitions, the sphere $S$ represents the homology class $A \in H_{2}(\tilde{M})$ and Theorem 6.2 follows.

\section{Graphs of trees and surfaces in normal form}

In this section, we give a description of a sphere in normal form in $M$. In the well studied case of normal surfaces in three-manifolds, a normal surface is described by normal coordinates, which are just the number of pieces of each form. However normal spheres are more complicated. We give a description of these in terms of the so-called graph of trees, which is analogous to a graph of groups.

\subsection{Graphs of trees}

Let $\Gamma$ be the dual graph to the decomposition of $M$ by standard spheres, with pairs $e$ and $\bar{e}$ of edges of $\Gamma$ corresponding to standard spheres $\Sigma_{i}$ and vertices to components $P_{k}$ of $M^{*}$. 
More precisely, there is a vertex $v_{k}$ corresponding to each component $P_{k}$ of $M^{*}$. For each sphere $\Sigma_{i}$ and component $P_{k}$ with $\Sigma_{i}$ a boundary component of $P_{k}$, there is an edge $e$ with initial vertex $o(e)=v$ and terminal vertex $t(e)$ the vertex corresponding to the other component containing $\Sigma_{i}$ (which may also be $P_{k}$ ), as well as another edge $\bar{e}$ with $(o(\bar{e}), t(\bar{e}))=(t(e), o(e))$. Note that each vertex of $\Gamma$ is trivalent.

Recall that a graph of groups for such a decomposition consists of groups $G(v), G(e)$ associated to vertices and edges of $\Gamma$ respectively, together with isomorphisms $G(e) \rightarrow$ $G(\bar{e})$ and for each edge $e \in E(\Gamma)$ an injective homomorphism from $G(e)$ to $G(o(e))$. By taking compositions, we also get an inclusion map from $G(e)$ to $G(t(e))$.

A graph of trees is analogous. More precisely, a graph of trees associated to $\Gamma$ consists of a non-empty, finite, oriented tree $t(e)$ associated to each edge $e \in E(\Gamma)$, a non-empty, oriented, finite tree $t(v)$ associated to each vertex $v \in V(\Gamma)$, and for each edge $e$ of $\Gamma$ an isomorphism from $t(e)$ to $t(\bar{e})$ and an injective orientation preserving simplicial map from $t(e)$ to $t(o(e))$.

We shall use more intuitive terminology for graphs and trees, and refer to the pair of oriented edges $e, \bar{e}$ as a single edge of the graph with adjoining vertices $o(e)$ and $t(e)$. By taking compositions if necessary, we have maps from the tree $t(e)$ to the tree $t(v)$ for $v$ a vertex of $e$.

If $P_{k}$ is the component corresponding to a vertex $v$ of $\Gamma$, we shall generally refer to $t(v)$ as $t\left(P_{k}\right)$. We similarly refer to the tree $t(e)$ as $t\left(\Sigma_{i}\right)$ if $\Sigma_{i}$ is the corresponding standard sphere.

\subsection{Normal spheres and graphs of trees}

Let $S$ be a normal sphere with a fixed co-orientation. We associate to $S$ a graph of trees structure. We remark that the construction applies to any surface in $M$ transversal to the standard sphere whose intersection with each component $P_{k}$ is of the standard form of a normal sphere. We shall call such a surface a normal surface. We shall assume that normal surfaces have fixed co-orientations.

7.2.1 Trees associated to the edges of $\Gamma$. Let $\Sigma_{i}$ be a standard sphere. Then, $S \cap \Sigma_{i}$ is a collection of disjoint circles. Consider the graph $t\left(\Sigma_{i}\right)$ whose edges $e$ correspond to the circles of intersection and vertices $v$ to complementary components, with $v$ a vertex of $e$ if the boundary of the component corresponding to $v$ contains the circle corresponding to $e$. The co-orientation of $S$ induces co-orientations for each of the circles of intersection. This gives an orientation for the edges of the graph $t\left(\Sigma_{i}\right)$.

Note that this construction associates a graph $t(\mathcal{C})$ to any collection $\mathcal{C}$ of disjoint co-oriented circles in the sphere. Further, that there is an embedding of $t(\mathcal{C})$ in the sphere that makes it dual to the decomposition of the sphere by the given circles.

Lemma 7.1. The graph $t(\mathcal{C})$ associated to a collection of disjoint co-oriented circles $\mathcal{C}$ in the sphere is a non-empty finite oriented tree. Conversely, any non-empty finite oriented tree $t$ is isomorphic to $t(\mathcal{C})$ for some collection of disjoint circles $\mathcal{C}$ in the sphere. Furthermore, the collection associated to a given tree is unique up to isotopy.

Proof. First, we shall prove that the graph $t=t(\mathcal{C})$ is a tree. If $t$ is not connected, then we associate to each component $t^{\prime}$ of $t$ the union of the circles corresponding to the edges in $t^{\prime}$ and the complementary components corresponding to the vertices in $t^{\prime}$. By construction this is an open subset of the sphere, for, if an edge (which corresponds to a circle) lies in $t^{\prime}$ so 
do its adjoining vertices (which correspond to the components containing the circle). Thus, we get a non-trivial partition of the sphere into open sets corresponding to components of $t$, which is impossible as the sphere is connected. Hence $t$ has only one component.

Further, given an edge $e$ of $t$, the corresponding circle $C$ separates the sphere $S$ into two components. It is easy to see that $t-\operatorname{int}(e)$ also consists of two components, corresponding to the components of $S-C$. Thus, every edge in $t$ separates. As $t$ is connected, it follows that $t$ is a tree. The tree $t$ is non-empty as there is at least one component in the complement of $\mathcal{C}$.

We prove the converse by induction on the number of vertices of $t$. The tree consisting of a single vertex corresponds to the empty collection of circles. Let $t$ be a finite tree and assume that the result holds for all trees with fewer vertices. Let $v$ be a terminal vertex of $t$ and $e$ the adjoining edge. Consider the subtree $t^{\prime}=t \backslash(v \cup \operatorname{int}(e))$ of $t$. Let $v^{\prime} \in t^{\prime}$ be the vertex of $e$ distinct from $v$.

By the induction hypothesis, there is a collection $\mathcal{C}^{\prime}$ of co-oriented circles whose associated tree is $t^{\prime}$. The vertex $v^{\prime}$ corresponds to some component $V^{\prime}$ of the complement of the circles $\mathcal{C}$. Let $\mathcal{C}$ be obtained from $\mathcal{C}^{\prime}$ by adding a circle which is the boundary of a disc in $V^{\prime}$. It is easy to see that, on choosing the appropriate co-orientation for this circle, the associated tree is $t$.

Uniqueness follows similarly by induction. Namely, we can assume that the circles in the collection $\mathcal{C}^{\prime}$ are unique up to isotopy. As $v$ is a terminal vertex, the circle in $\mathcal{C} \backslash \mathcal{C}^{\prime}$ must bound a disc in $V^{\prime}$. As any two discs in a connected surface are isotopic, it follows that $\mathcal{C}$ is unique up to isotopy.

We associate oriented trees to the edges of $\Gamma$ by taking the oriented trees associated to the corresponding standard spheres $\Sigma_{i}$.

7.2.2 Trees associated to vertices of $\Gamma$. Let $v$ be a vertex of $\Gamma$ corresponding to the component $P_{k}$ of $M^{*}$. Let $\mathcal{P}$ be a collection of pieces in $P_{k}$, i.e., a disjoint collection of pairs of pants, cylinders and discs satisfying the conditions for normal spheres.

Let $e_{1}, e_{2}$ and $e_{3}$ be the edges adjoining $v$ and let $\Sigma_{i}$ be the corresponding boundary components. Then, $\mathcal{C}_{i}=\Sigma_{i} \cap \mathcal{P}$ is a collection of disjoint circles in $\Sigma_{i}$, to which we can associate a tree $t_{i}=t\left(e_{i}\right)$ as in Lemma 7.1. We shall construct a tree $t=t(\mathcal{P})$ and inclusion maps from the trees $t_{i}$ to $t(v)$.

The pieces $\mathcal{P}$ form a collection of co-oriented co-dimension 1 proper submanifolds in the simply-connected manifold $P_{k}$. Let $t=t(\mathcal{P})$ be the dual graph to this decomposition namely, we take an edge for each piece and a vertex in each complementary component, with the edges intersecting the pieces transversally in a single point.

\section{Lemma 7.2. The graph $t$ is a tree.}

Proof. The proof is essentially the same as the first part of Lemma 7.1.

Firstly, we can associate to each component $t^{\prime}$ of $t$ an open set consisting of the union of the pieces corresponding to edges of $t^{\prime}$ and complementary components consisting of vertices of $t^{\prime}$. This gives a decomposition of $P_{k}$ as a union of open sets. As $P_{k}$ is connected, it follows that $t$ is connected.

Next, an edge $e$ of $t$ corresponds to a piece in $P_{k}$. As $P_{k}$ is simply-connected, the piece partitions $P_{k}$ into two components. This gives a corresponding decomposition of $t-\operatorname{int}(e)$ into two components. Thus, each edge of $t$ separates, showing that $t$ is a tree. 
We have natural inclusion maps from $t_{i}$ to $t$ - namely, each component of $\Sigma_{i}-\mathcal{C}_{i}$ is contained in a unique component of $P_{k}-\mathcal{P}$ giving a natural map of vertices. Similarly, each component of $\mathcal{C}_{i}$ is contained in a unique component of $\mathcal{P}$, giving a map of edges. It is clear that these maps give an injective simplicial map. If $S \cap P_{k}=\phi$, then $t(v)$ contains only one vertex and no edges and we have natural inclusion maps.

The next step in our construction is to show that trees $t_{i}$ and $t$ satisfying certain conditions are associated to collections of pieces. We fix a vertex $v$ of $\Gamma$ corresponding to the component $P_{k}$. Let $\Sigma_{i}, i=1,2,3$ be the corresponding boundary components of $P_{k}$ and let $t_{i}$ be the corresponding trees.

Lemma 7.3. Let $\mathbb{T}$ and $\mathbb{T}_{j}, 1 \leq j \leq 3$ be non-empty finite trees. Let $i_{j}: \mathbb{T}_{j} \rightarrow \mathbb{T}$ be injective homomorphisms satisfying the following conditions.

(1) The union of the images of the maps $i_{j}$ is $\mathbb{T}$.

(2) If an edge e $\subset \mathbb{T}$ is in the image of only one inclusion map $i_{j}$, say $i_{1}$, then the images of $i_{2}$ and $i_{3}$ are contained in opposite components of $\mathbb{T}-\operatorname{int}(e)$.

Then, there is a collection of pieces $\mathcal{P}$ so that the associated trees and inclusion maps are the given ones.

Remark 7.4. The conditions correspond to $\mathcal{P}$ having no closed components and to discs in $\mathcal{P}$ separating the two boundary spheres of the component $P_{k}$ that they do not intersect. It is thus easy to see that trees associated to a normal surface satisfy these conditions.

\section{DEFINITION 7.5}

We say that a graph of trees is regular if it satisfies conditions 1 and 2 of Lemma 7.3 for each vertex of $\Gamma$.

Proof of Lemma 7.3. We proceed by induction on the number of vertices in $\mathbb{T}$. The base case, where the tree $\mathbb{T}$ consists of a single vertex (and hence so do the trees $\mathbb{T}_{j}$ ) corresponds to the empty collection of pieces.

Assume that the result holds for all trees with fewer vertices than $\mathbb{T}$. Let $v$ be a terminal vertex of $\mathbb{T}$ and let $e$ be the unique adjoining edge. Let $v^{\prime}$ be the other vertex of $e$.

We first construct trees $t_{j}$ and $t$ with fewer vertices satisfying the hypothesis. The tree $t$ is obtained from $\mathbb{T}$ by deleting the vertex $v$ and the interior of the edge $e$. If the edge $e$ is in the image of $i_{j}$, the tree $t_{j}$ is obtained from $\mathbb{T}_{j}$ by deleting the interior of $i_{j}^{-1}(e)$ and the vertex $i_{j}^{-1}(v)$. Otherwise $t_{j}=\mathbb{T}_{j}$. More intuitively, we delete an innermost piece and all its boundary components.

It is easy to see that the trees $t$ and $t_{j}, j=1,2,3$, satisfy the hypothesis of the lemma, except that one or two of the trees $t_{j}$ may be empty, which happens if and only if the corresponding tree $\mathbb{T}_{j}$ consists of a single vertex mapping to $v$. In this case, we replace the empty tree $t_{j}$ by a single vertex, which we map to $v^{\prime}$ under the inclusion map $i_{j}$.

By the induction hypothesis, we can construct a collection of pieces $\mathcal{Q}$ with $t$ and $t_{j}$ as the corresponding trees with the given inclusion maps. We shall add a single piece, corresponding to $e$, to obtain the required collection of pieces $\mathcal{P}$.

Note that by condition 1 , the edge $e$ is the image of edges in 1,2 or 3 trees $\mathbb{T}_{j}$. We shall add a disc, annulus or a pair of pants according to the number of such trees.

First, consider the case when $e$ lies in the image of only one tree, say $\mathbb{T}_{1}$. By condition 2 , it follows that the image of one of the other trees $\mathbb{T}_{j}$, say $\mathbb{T}_{2}$, consists of the single 
vertex $v$. It follows that the sphere $\Sigma_{2}$ is disjoint from the collection $\mathcal{Q}$. Further, by the above construction, the tree $t_{2}$ is a single vertex mapping to $v^{\prime}$, hence the sphere $\Sigma_{2}$ is in the component corresponding to $v^{\prime}$. As $v^{\prime}$ is also in the image of $\mathbb{T}_{1}$, the component corresponding to $v^{\prime}$ intersects $\Sigma_{1}$. Hence there is an embedded arc $\gamma$ disjoint from $\mathcal{Q}$ joining $\Sigma_{2}$ to $\Sigma_{1}$.

We consider the disc $D$ that is the boundary of a regular neighbourhood of $\gamma \cup \Sigma_{2}$. By construction this is disjoint from $\mathcal{Q}$. It is easy to see that the collection $\mathcal{P}$ consisting of $\mathcal{Q}$ together with the disc $D$ corresponds to the trees $\mathbb{T}$ and $\mathbb{T}_{j}$.

We now turn to the other two cases. Firstly, if $e$ is in the image of two trees, say $\mathbb{T}_{1}$ and $\mathbb{T}_{2}$, then $v^{\prime}$ is in the image of both these trees. Hence the component $V^{\prime}$ corresponding to $v^{\prime}$ intersects both $\Sigma_{1}$ and $\Sigma_{2}$. Hence there is an embedded arc in $V^{\prime}$ joining $\gamma_{1}$ to $\gamma_{2}$. The boundary of a regular neighbourhood of this arc gives an annulus $A$ disjoint from $\mathcal{Q}$. The union of $\mathcal{Q}$ with the annulus $A$ gives the required collection $\mathcal{P}$.

The case when $e$ is in the image of all three trees $\mathbb{T}_{j}$, and hence the component $V^{\prime}$ corresponding to $v^{\prime}$ intersects each $\Sigma_{j}$, is similar. We take a point in the interior of $V^{\prime}$ and join it by disjoint embedded arcs to the spheres $\Sigma_{j}$, giving an embedding of a tripod $\tau$. A regular neighbourhood of $\tau$ gives a pair of pants. The union of $\mathcal{Q}$ with this pair of pants gives the required collection $\mathcal{P}$ of pieces.

We now see that we can construct a surface in normal form corresponding to the given graph of trees. This is obtained by gluing together the pieces.

Lemma 7.6. A regular graph of trees is the graph of trees associated to a surface in normal form.

Proof. We consider first the manifold $\hat{M}$ obtained from $M$ by deleting regular neighbourhoods of the surfaces $\Sigma_{i}$. The components of $\hat{M}$ can be identified with the components $P_{k}$ of $M^{*}$. We assume that such identifications have been made.

By Lemma 7.3, there are pieces in each such component $P_{k}$ which correspond to the given graph of trees. The normal surface we construct is the union of these pieces with annuli in the neighbourhoods $\Sigma_{i} \times[-1,1]$ of the surfaces $\Sigma_{i}$.

Consider the neighbourhood $\Sigma_{i} \times[-1,1]$ of such a surface $\Sigma_{i}$. The boundary of the pieces in the components $P_{k}$ give a collection of circles in the components $\Sigma_{i} \times\{-1,1\}$ of the boundary of $\hat{M}$, which can be identified with $\Sigma_{i}$.

These collections of circles correspond, under Lemma 7.1, with the tree associated with $\Sigma_{i}$. By the uniqueness statement of Lemma 7.1, these collections of circles are isotopic. It follows that there is a family of disjoint annuli in $\Sigma_{i} \times[-1,1]$ with boundaries the given families of disjoint circles in $\Sigma_{i} \times\{-1,1\}$.

The union of the pieces in the components of $\hat{M}$ together with the annuli in the neighbourhoods of the surfaces $\Sigma_{i}$ is thus a (not necessarily connected) surface. It is clear that this corresponds to the given graph of trees.

\section{Embedding classes in $M$}

We now give a constructive proof of the main result of [9] giving a criterion for a class $A \in \pi_{2}(M)=H_{2}(\tilde{M})$ to be representable by an embedded sphere in $M$.

Theorem 8.1. The class $A \in \pi_{2}(M)$ can be represented by an embedded sphere in $M$ if and only if $A$ can be represented by an embedded sphere in $\tilde{M}$ and, for all deck transformations, $g \in \pi_{1}(M)$, A and $g A$ do not cross. 
If $A$ can be represented by an embedded sphere $S$ in $M$, then one can easily see that a lift $\tilde{S}$ and all of its translates in $\tilde{M}$ are disjoint. Therefore, $A$ and $g A$ can be represented by disjoint embedded spheres in $\tilde{M}$, for all $g \in \pi_{1}(M)$, which implies $A$ and $g A$ do not cross.

The rest of this section is devoted to a constructive proof of the converse. Let $A$ be a class in $\pi_{2}(M)$ that satisfies the hypothesis. We shall associate to the class $A$ a regular graph of trees, hence a surface in normal form. We then show that this normal surface in $A$ is the homeomorphic image under the restriction of the covering map of a sphere in $\tilde{M}$ representing the class $A$. It follows that the normal surface constructed is a sphere and represents the class $A$.

\subsection{Trees and Dunwoody's construction}

We construct the trees associated to vertices and edges of $\Gamma$ using the characterization of trees in terms of their edges with an ordering due to Dunwoody. We recall some definitions and results from [8] and give a simple extension that we need.

We shall require precise combinatorial descriptions of graphs and associated objects. We recall these below.

DEFINITION 8.2 (see Definition 2.3)

A graph $\mathbb{T}$ consists of two sets $E(\mathbb{T})$ and $V(\mathbb{T})$, called the edges and vertices of $\mathbb{T}$, a mapping from $E(\mathbb{T})$ to $E(\mathbb{T})$ denoted $e \mapsto \bar{e}$, for which $e \neq \bar{e}$ and $\overline{\bar{e}}=e$, and a mapping from $E(\mathbb{T})$ to $V(\mathbb{T}) \times V(\mathbb{T})$, denoted $e \mapsto(o(e), t(e))$, such that $\bar{e} \mapsto(t(e), o(e))$ for every $e \in E(\mathbb{T})$.

\section{DEFINITION 8.3}

An edge path in $\mathbb{T}$ is a sequence $e_{1}, e_{2}, \ldots, e_{n}$ of edges, such that $t\left(e_{i}\right)=o\left(e_{i+1}\right)$, $e_{i} \neq \bar{e}_{i+1}$, for $i=1,2, \ldots, n-1$.

If $e, f \in E(\mathbb{T})$, we write $e \leq f$ if there is an edge path $e_{1}, e_{2}, \ldots, e_{n}$ for which $e_{1}=e$ and $e_{n}=f$. If $\mathbb{T}$ is a tree, then $\leq$ determines a partial ordering on $E(\mathbb{T})$. In addition the following conditions are satisfied:

(1) if $e \leq f$, then $\bar{f} \leq \bar{e}$;

(2) if $e \leq f$, there are only finitely many $g$ for which $e \leq g \leq f$;

(3) for any pair $e, f$, at least one of $e \leq f, e \leq \bar{f}, \bar{e} \leq \bar{f}, \bar{e} \leq \bar{f}$ holds;

(4) for no pair $e, f$ is $e \leq f$ and $e \leq \overline{\bar{f}}$;

(5) for no pair $e, f$ is $e \leq f$ and $\bar{e} \leq f$.

Theorem 8.4 (Dunwoody). Let $(E, \leq)$ be a partially ordered set with a mapping $E \rightarrow E$, $e \rightarrow \bar{e}$, for which $e=\overline{\bar{e}}$, and suppose the above conditions (1)-(5) are satisfied. Then, there exists a tree $\mathbb{T}$ with $E=E(\mathbb{T})$, where $E(\mathbb{T})$ is the set of edges of $\mathbb{T}$ and the order relation on $E$ is precisely that determined by edge paths in $\mathbb{T}$ as above.

Remark 8.5. The vertices of the tree in the above theorem correspond to equivalence classes of edges, with two edges $e$ and $f$ equivalent if $e$ and $\bar{f}$ are adjacent with $e \leq \bar{f}$. Here, we say edges $e_{1}$ and $e_{2}$, with $e_{1} \leq e_{2}$, are adjacent if whenever $e_{1} \leq g \leq e_{2}$, then $g=e_{1}$ or $g=e_{2}$. 
Intuitively, the equivalence relation in the above construction corresponds to the terminal vertices of edges being the same. There is an exceptional case, when the set of edges is empty. In this case the tree consists of a single vertex (even though there are no equivalence classes).

\section{DEFINITION 8.6}

We say that an edge $f$ is on the positive side of $e$ if either $e \leq f$ or $e \leq \bar{f}$. Otherwise we say that $f$ is on the negative side of $e$.

The following is immediate from Dunwoody's conditions.

Lemma 8.7. The edge $f$ is on the negative side of $e$ if and only if $f$ is on the positive side of $\bar{e}$.

As a simple extension to Dunwoody's result, we obtain the following.

Lemma 8.8. Let $B_{1}, \ldots, B_{n}$ be homology classes in $H_{2}(\tilde{M})$ so that no pair crosses and $B_{i} \neq \pm B_{j}$ for $1 \leq i<j \leq n$. Then, there is an associated oriented tree with edges $e_{i}$ corresponding to classes $B_{i}$ so that $e_{j}$ is on the positive side of $e_{i}$ if and only if $B_{j}$ is on the positive side of $B_{i}$.

Proof. We define the set $E$ of edges to be $\left\{B_{1}, \ldots, B_{n},-B_{1}, \ldots,-B_{n}\right\}$. We define $B_{i} \leq$ $B_{j}$ if $E^{+}\left(B_{j}\right) \subset E^{+}\left(B_{i}\right)$. We can easily check ' $\leq$ ' turns out be a partial order relation satisfying the hypothesis of Theorem 8.4 . The result follows.

\subsection{Graph of trees structure associated to the homology class A}

We now associate a regular graph of trees to a class $A$ satisfying the hypothesis of Theorem 8.1, and hence construct a corresponding normal sphere $S$ in $M$ representing the class $A$. Our construction is motivated by the observations in [10] (see Lemma 5.4) that, for an embedded sphere $S$ in $M$ in normal form and a standard sphere $\Sigma_{i}$ in $M$, the components of $S \cap \Sigma_{i}$ are in bijective correspondence with translates of a lift of $S$ that cross a lift $\tilde{\Sigma}_{i}$ of $\Sigma_{i}$.

As we have seen, the homology class $A$ of a sphere $S$ in $\tilde{M}$ determines the corresponding partition of ends of $\tilde{M}$, and hence crossing. Further, the order on edges obtained as in Lemma 8.8 correspond to on which side of a translate $g A$ of $A$ another translate $h A$ lies. This is, by definition, determined by the partition corresponding to $A$, and hence the homology class of the sphere $S$.

Consider now a homology class satisfying the hypothesis of Theorem 8.1 and its associated partition. We shall associate a graph of trees to $A$.

Observe that, as $A$ is disjoint from all its translates, any finite collection of translates of $A$ satisfies the hypothesis of Lemma 8.8. This follows from the remarks following Definition 4.1. We shall use this observation repeatedly.

8.2.1 Tree associated to edges of $\Gamma$. Let $\Sigma_{i}$ be a standard sphere corresponding to an edge $e \in E(\Gamma)$. We consider the set of pairs $\left(\tilde{\Sigma}_{i}, g A\right)$ with $\tilde{\Sigma}_{i}$ a lift of $\Sigma_{i}$ so that $g A$ crosses $\tilde{\Sigma}_{i}$. On the set of such pairs, we introduce the equivalence relation given by

$$
\left(\tilde{\Sigma}_{i}, g A\right) \sim\left(h \tilde{\Sigma}_{i}, h g A\right), h \in \pi_{1}(M)
$$

with $h \in \pi_{1}(M)$ acting as a deck transformation. 
Let $E\left(\Sigma_{i}\right)$ be the set of equivalence classes under this equivalence relation. As two lifts are related by a deck transformation, for each fixed lift $\tilde{\Sigma}_{i}^{0}$, there is a unique representative in each equivalence class of the form $\left(\tilde{\Sigma}_{i}^{0}, g A\right)$. By proper discontinuity of the action by deck transformations, as homology classes are compactly supported, there are only finitely many equivalence classes in $E\left(\Sigma_{i}\right)$. We also observe that each equivalence class has a unique representative of the form $\left(\tilde{\Sigma}_{i}, A\right)$.

Fix a lift $\tilde{\Sigma}_{i}^{0}$ of $\Sigma_{i}$. Then, as above, the equivalence classes correspond to the finitely many homology classes $g A$ so that $g A$ crosses $\tilde{\Sigma}_{i}^{0}$. We let $t(e)=t\left(\Sigma_{i}\right)$ be a tree obtained using Lemma 8.8 corresponding to the homology classes $g A$ that cross $\tilde{\Sigma}_{i}$. Thus, the unoriented edges of $t\left(\Sigma_{i}\right)$ correspond to equivalence classes of pairs $\left(\tilde{\Sigma}_{i}, g A\right)$ which cross, i.e., elements of $E\left(\Sigma_{i}\right)$.

We observe that if $h \tilde{\Sigma}_{i}^{0}$ is another lift, then the order obtained from this lift is the same as that obtained from $\tilde{\Sigma}_{i}^{0}$, as the homology classes crossing $h \tilde{\Sigma}_{i}^{0}$ are obtained from those crossing $\tilde{\Sigma}_{i}^{0}$ by applying the deck transformation $h$, which preserves the order. We associate the tree $t\left(\Sigma_{i}\right)$ to the edge $e$ of $\Gamma$ corresponding to $\Sigma_{i}$.

8.2.2 Tree associated to vertices of $\Gamma$. Similarly, for a component $P_{k}$ of $M^{*}$ corresponding to a vertex $v \in \Gamma$, we consider a lift $\tilde{P}_{k} \subset \tilde{M}^{*}$ of $P_{k}$, which we recall is homeomorphic to a 3 -sphere with the interiors of three balls deleted. We say that $g A$ crosses $\tilde{P}_{k}$ if and only if it crosses one of the boundary components (which are standard spheres) of $\tilde{P}_{k}$.

We once more consider pairs $\left(\tilde{P}_{k}, g A\right)$ so that $g A$ crosses $\tilde{P}_{k}$, under the equivalence relation $\left(\tilde{P}_{k}, g A\right) \sim\left(h \tilde{P}_{k}, h g A\right)$ with $h \in \pi_{1}(M)$. As in the case of the trees $t\left(\Sigma_{i}\right)$, we obtain a tree $t(v)=t\left(P_{k}\right)$ with the set of unoriented edges the set $E\left(P_{k}\right)$ of such equivalence classes using Lemma 8.8. Note that we once more obtain a unique equivalence class on fixing either a lift $\tilde{P}_{k}^{0}$ of $P_{k}$ or by considering pairs of the form $\left(\tilde{P}_{k}, A\right)$.

8.2.3 The inclusion maps. For a component $P_{k}$ of $M^{*}$ and a boundary component $\Sigma_{i}$ of $P_{k}$, we choose and fix lifts $\tilde{P}_{k}^{0}$ and $\tilde{\Sigma}_{i}^{0}$ so that $\tilde{\Sigma}_{i}^{0}$ is a boundary component of $\tilde{P}_{k}^{0}$.

As we have observed, each edge in the tree $t\left(\Sigma_{i}\right)$ has a unique representative of the form $\left(\tilde{\Sigma}_{i}^{0}, g A\right)$. We map this edge of $t\left(\Sigma_{i}\right)$ to the edge corresponding to $\left(\tilde{P}_{k}^{0}, g A\right)$ in $t\left(P_{k}\right)$. Observe that by definition $g A$ crosses $\tilde{P}_{k}^{0}$, so this map on edges makes sense.

We now extend the map to vertices. In Dunwoody's construction (see Remark 8.5), vertices correspond to equivalence classes of edges. We see, using the following lemma, that $i_{j}$ gives a well-defined (injective) map on vertices.

Lemma 8.9. Let $A_{i}, A_{j}$ and $A_{k}$ be translates of $A$ such that $A_{i} \leq A_{j} \leq A_{k}$. If $A_{i}$ and $A_{k}$ cross the homology class of the boundary sphere $\tilde{\Sigma}_{i}$ of $\tilde{P}_{k}$, so does $A_{j}$.

Proof. As $A_{j} \leq A_{k}, E^{+}\left(A_{k}\right) \subset E^{+}\left(A_{j}\right)$. As $A_{k}$ crosses the homology class $\left[\tilde{\Sigma}_{i}\right]$ of the boundary sphere $\tilde{\Sigma}_{i}$, we have $E^{ \pm}\left(\left[\Sigma_{i}\right]\right) \cap E^{+}\left(A_{k}\right) \neq \phi$. This implies $E^{ \pm}\left(\left[\Sigma_{i}\right]\right) \cap$ $E^{+}\left(A_{j}\right) \neq \phi$.

As $A_{i} \leq A_{j}, E^{+}\left(A_{j}\right) \subset E^{+}\left(A_{i}\right)$ and hence, $E^{-}\left(A_{i}\right) \subset E^{-}\left(A_{j}\right)$. As $A_{i}$ crosses the homology class $\left[\tilde{\Sigma}_{i}\right]$ of the boundary sphere $\tilde{\Sigma}_{i}$, we have $E^{ \pm}\left(\left[\Sigma_{i}\right]\right) \cap E^{-}\left(A_{i}\right) \neq \phi$. This implies $E^{ \pm}\left(\left[\Sigma_{i}\right]\right) \cap E^{-}\left(A_{j}\right) \neq \phi$.

Thus, all the four intersections $E^{ \pm}\left(\left[\Sigma_{i}\right]\right) \cap E^{ \pm}\left(A_{j}\right)$ are non-empty. Hence, $A_{j}$ crosses the homology class of the boundary sphere $\widetilde{\Sigma_{i}}$ of $\widetilde{P}_{k}$. 


\section{COROLLARY 8.10}

If $e$ and $f$ are adjacent edges in $t\left(\Sigma_{j}\right)$, then their images are adjacent edges in $t\left(P_{k}\right)$.

Proof. Suppose $e$ and $f$ correspond to the homology classes $A_{i}$ and $A_{k}$, oriented so that $A_{i}<A_{k}$. If their images are not adjacent in $t\left(P_{k}\right)$, then there is a class $A_{j}$ with $A_{i}<A_{j}<$ $A_{k}$ corresponding to an edge in between the images of $e$ and $f$. But, by Lemma 8.9, $A_{j}$ crosses $\Sigma_{j}$, and hence gives an edge of $t\left(\Sigma_{j}\right)$ between $e$ and $f$, contradicting the hypothesis that $e$ and $f$ are adjacent. Hence, the images of $e$ and $f$ in $t\left(P_{k}\right)$ must be adjacent.

Thus, if edges $e$ and $f$ are equivalent in $t\left(\Sigma_{j}\right)$, their images are also equivalent in $t\left(P_{k}\right)$. It follows that, if the set of edges of $t\left(\Sigma_{i}\right)$ is non-empty, we have well-defined maps on vertices, giving simplicial inclusions $i_{j}$ from $t\left(\Sigma_{j}\right)$ to $t\left(P_{k}\right)$.

We finally turn to the case when the set of edges of $t\left(\Sigma_{j}\right)$ is empty. In this case $t\left(\Sigma_{j}\right)$ consists of a single vertex and $\Sigma_{j}$ does not cross any translate of the class $A$. We can thus apply Lemma 8.8 to the union of the translates of $A$ that cross $\tilde{P}_{k}$ with the class represented by the lift $\tilde{\Sigma}_{j}$ of $\Sigma_{j}$ in the boundary of $\tilde{P}_{k}$ to obtain a tree $t^{\prime}$ which is the union of $t\left(P_{k}\right)$ and a single edge $e$ corresponding to $\tilde{\Sigma}_{j}$.

\section{Lemma 8.11. At least one of the vertices of $e$ is a terminal vertex of $t^{\prime}$.}

Proof. Suppose not. Then, there are translates $g_{1} A$ and $g_{2} A$ of $A$ on opposite sides of the sphere $\tilde{\Sigma}_{j}$. If $\tilde{\Sigma}_{i}$ is another boundary sphere of $\tilde{P}_{k}$, then by Lemma $4.3, g_{1} A$ and $g_{2} A$ cannot both cross $\tilde{\Sigma}_{i}$. Thus, the translates $g_{1} A$ and $g_{2} A$ cross the other two boundary components of $\tilde{P}_{k}$. By Lemma 4.3 , it follows that the other two boundary components of $\tilde{P}_{k}$ must be on opposite sides of $\Sigma_{j}$, which is clearly not the case. This gives the required contradiction.

If both vertices of $e$ are terminal vertices, then $t\left(P_{k}\right)$ is a tree containing only one vertex and no edges, so there is only one possible map from $t\left(\Sigma_{j}\right)$. Otherwise, let $v$ be the terminal vertex of $e$ and $v^{\prime}$ the other vertex. As $t^{\prime}$ is connected, it is easy to see that $v^{\prime} \in t$. We define the inclusion map as mapping $t\left(\Sigma_{j}\right)$, which is a single vertex, to $v^{\prime}$.

\section{Lemma 8.12. The graph of trees associated to the class $A$ is regular.}

Proof. Let $v$ be a vertex of $\Gamma$ corresponding to the component $P_{k}$ of $M^{*}$. Let $e_{1}, e_{2}$ and $e_{3}$ be the edges adjoining $v$ and let $\Sigma_{i}$ be the corresponding boundary components of $P_{k}$. We can easily see that the union of the images of the maps $i_{j}: t\left(\Sigma_{i}\right) \rightarrow t\left(P_{k}\right)$ is $t\left(P_{k}\right)$.

Now suppose that there is an edge $e$ in $t\left(P_{k}\right)$ such that $e$ is in the image of only one inclusion map $i_{j}$, say $i_{1}$. Let $\tilde{\Sigma}_{1}$ be a lift of $\Sigma_{1}$ and $\tilde{P}_{k}$ of $P_{k}$ such that $\tilde{\Sigma}_{1}$ is boundary sphere of $\tilde{P}_{k}$. Let $\tilde{\Sigma}_{2}$ and $\tilde{\Sigma}_{3}$ be the other boundary spheres of $\tilde{P}_{k}$ which are lifts of $\Sigma_{2}$ and $\Sigma_{3}$ respectively. Then, corresponding to the edge $e$ of $t\left(P_{k}\right)$, there is a translate $g A$ of $A$ such that $g A$ crosses $\tilde{\Sigma}_{1}$. Note that $g A$ does not cross $\tilde{\Sigma}_{2}$ and $\tilde{\Sigma}_{3}$. By Lemma 5.6, one of the spheres $\tilde{\Sigma}_{1}$ and $\tilde{\Sigma}_{2}$ is on the positive side of $g A$ and the other on the negative side. Suppose that the sphere $\tilde{\Sigma}_{2}$ is on the positive side and $\tilde{\Sigma}_{3}$ is on the negative side of $g A$. By Lemma 4.3, any translate of $A$ which crosses $\tilde{\Sigma}_{2}$ is on the positive side of $g A$ and any translate of $A$ which crosses $\tilde{\Sigma}_{3}$ is on the negative side of $g A$. This shows that image of every edge of $t\left(\Sigma_{2}\right)$ under the inclusion map $i_{2}$ (hence, the subtree $i_{2}\left(t\left(\Sigma_{2}\right)\right)$ of $\left.t\left(P_{k}\right)\right)$ lies on the positive side of the edge $e$ and image of every edge of $t\left(\Sigma_{3}\right)$ under the inclusion map $i_{3}$ (hence, the subtree $i_{3}\left(t\left(\Sigma_{3}\right)\right)$ of the tree $\left.t\left(P_{k}\right)\right)$ lies on the negative side of $e$. If the 
tree $t\left(\Sigma_{2}\right)$ is a tree containing only one vertex and no edges, the inclusion map $i_{2}$ maps this vertex to a vertex in $t\left(P_{k}\right)$ on the positive side of $e$ as $\tilde{\Sigma}_{2}$ is on the positive side of $g A$. Similarly, if the tree $t\left(\Sigma_{3}\right)$ is a tree containing only one vertex and no edges, the inclusion map $i_{3}$ maps this vertex to a vertex in $t\left(P_{k}\right)$ on the negative side of $e$. Thus, the images of $i_{2}$ and $i_{3}$ are contained in opposite components of $t\left(P_{k}\right)-\operatorname{int}(e)$. This shows that the graph of trees associated to the class $A$ is regular.

8.2.4 Lift to the universal cover. We have associated to the homology class $A$ a regular graph of trees, and hence a surface $S$ in normal form by Lemma 7.6. We shall show that $S$ is a sphere and represents the class $A$. This follows from the following lemma.

Lemma 8.13. There is a sphere $\tilde{S} \subset \tilde{M}$ which represents the class $A$ and which maps homeomorphically to $S$ under the restriction of the covering map.

Proof. As in Lemma 5.9, we can associate a finite tree $\tau \subset T$ to $A$ with additional data that determines the class $A$. In Lemma 5.10, we showed that there is a normal sphere $\tilde{S}$ in $\tilde{M}$ that can be associated to the data, which is in the class of $A$. We shall see that $\tilde{S}$ can be chosen so that its image in $M$ is $S$ and the covering map is injective on $\tilde{S}$.

Consider a standard vertex $v$ of $\tau$ (i.e., $v$ a vertex that is bivalent in $T$ ). The vertex $v$ corresponds to a standard sphere $\tilde{\Sigma}_{i}$ in $\tilde{M}$, which crosses $A$ as $v$ is in $\tau$. Consider the image $\Sigma_{i}$ of $\tilde{\Sigma}_{i}$. The tree $t\left(\Sigma_{i}\right)$ associated to $\Sigma_{i}$ contains a unique edge corresponding to pair $\left(\tilde{\Sigma}_{i}, A\right)$, and hence $\Sigma_{i}$ contains a circle $C$ corresponding to this pair. We can choose $\tilde{S} \cap \tilde{\Sigma}_{i}$, which is a single circle, to be the lift $\tilde{C}$ of $C \subset \Sigma_{i}$ to $\tilde{\Sigma}_{i}$.

Similarly, for each non-standard vertex $v$ of $\tau$ (i.e., $v$ a vertex that is trivalent in $T$ ), we have a corresponding component $\tilde{P}_{k} \subset \tilde{M}$ with image a component $P_{k}$ in $M$. By definition, $A$ crosses $\tilde{P}_{k}$, so there is a piece $P$ in $P_{k}$ corresponding to the pair $\left(\tilde{P}_{k}, A\right)$. We consider the lift $\tilde{P}$ of this piece to $\tilde{P}_{k}$.

Suppose $\Sigma_{i}$ is a boundary component of $P_{k}$, with the lift $\tilde{\Sigma}_{i}$ of $\Sigma_{i}$ contained in the lift $\tilde{P}_{k}$ of $P_{k}$. Then, under the inclusion map of the graph of trees, the edge corresponding to the pair $\left(\tilde{\Sigma}_{i}, A\right)$ maps to the edge corresponding to the pair $\left(\tilde{P}_{k}, A\right)$. Hence, the corresponding circle $\tilde{C} \subset \tilde{\Sigma}_{i}$ is a component of the boundary of the piece $\tilde{P}$.

It follows that the piece $\tilde{P}$ in each component $\tilde{P}_{j}$ containing a standard sphere $\tilde{\Sigma}_{i}$ intersects $\tilde{\Sigma}_{i}$ in the circle $\tilde{C}$. Hence the union of the pieces constructed above give a surface.

Next, we see that the disc and cylinder pieces $\tilde{P}$ of $\tilde{S}$ correspond to the data determining $A$. This data is equivalent to specifying on which side of $\tilde{P}$ a boundary sphere $\tilde{\Sigma}_{i}$ of $\tilde{P}_{k}$ lies. Without loss of generality, assume that the sphere $\tilde{\Sigma}_{i}$ lies on the positive side of $A$. We shall show that $\tilde{\Sigma}_{i}$ also lies on the positive side of $\tilde{S}$. We have two cases, depending on whether or not another piece $P^{\prime}$ in $P_{k}$ has boundary on $\Sigma_{i}$.

If no such piece has boundary on $\Sigma_{i}$, then the tree associated to $\Sigma_{i}$ is a single vertex, which maps to a vertex in the tree $t\left(P_{k}\right)$ associated to $P_{k}$. Recall that this is the vertex $v^{\prime}$ that is a vertex of the new edge obtained by extending the tree $t\left(P_{k}\right)$ by adding the class $\left[\tilde{\Sigma}_{i}\right]$ to the translates of $A$. By construction, this lies on the positive side of the edge corresponding to the pair $\left(\tilde{P}_{k}, A\right)$ in $t\left(P_{k}\right)$ as $\tilde{\Sigma}_{i}$ lies on the positive side of $A$. As the surface $S$ is constructed using Lemma 7.3, the piece $P$ is thus embedded so that the sphere $\tilde{\Sigma}_{i}$ is on the positive side of $\tilde{S}$.

Suppose some piece $P^{\prime}$, corresponding to the crossing of $g A$ with $\tilde{P}_{k}$ has boundary on $\Sigma_{i}$. Observe that $g A$ and $\tilde{\Sigma}_{i}$ are on the same side of $A$, hence both on the positive side, otherwise we get a contradiction to Lemma 4.3. By construction of $S, P^{\prime}$ is on the positive side of $P$ in $P_{k}$, and hence $\tilde{\Sigma}_{i}$ is on the positive side of $\tilde{S}$, as required. 
Thus, by Lemma 5.9, the sphere $\tilde{S}$, represents the class $A$. As the pieces and circles in $\tilde{S}$ are lifts of those in $S$, and there is a one-to one correspondence between these, $S$ is the homeomorphic image of $\tilde{S}$ under the restriction of the covering.

As $\tilde{S}$ is a sphere representing the class $A$, so is $S$. This completes the proof of Theorem 8.1.

\section{References}

[1] Bestvina Mladen, Feighn Mark and Handel Michael, The Tits alternative for $\operatorname{Out}\left(F_{n}\right) \mathrm{I}$, Dynamics of exponentially-growing automorphisms, Ann. Math. (2) 151(2) (2000) 517-623

[2] Bestvina Mladen and Feighn Mark, The topology at infinity of $\operatorname{Out}\left(F_{n}\right)$, Invent. Math. 140(3) (2000) 651-692

[3] Bestvina M, Feighn M and Handel M, Laminations, trees, and irreducible automorphisms of free groups, Geom. Funct. Anal. 7(2) (1997) 215-244

[4] Bestvina Mladen and Handel Michael, Train tracks and automorphisms of free groups, Ann. Math. (2) 135(1) (1992) 1-51

[5] Bowditch Brian H, Intersection numbers and the hyperbolicity of the complex of curves, J. Reine Angew. Math. 598 (2006) 105129

[6] Culler M and Morgan J M, Group actions on R-trees, Proc. London Math. Soc. 55 (1987) 571-604

[7] Culler M and Vogtmann K, Modulii of graphs and automorphisms of free group, Invent. Math. 87(1) (1986) 91-119

[8] Dunwoody M J, Accessibility and groups of cohomological dimension one, Proc. London Math. Soc. (3) 38(2) (1979) 193-215

[9] Gadgil S, Embedded spheres in $\sharp_{n} S^{2} \times S^{1}$, Topology and its Applications 153 (2006) $1141-1151$

[10] Gadgil S and Pandit S, Algebraic and geometric intersection numbers for free groups, Topology and its Applications 156(9) (2009) 1615-1619

[11] Gadgil S and Pandit S, Geosphere Laminations for free groups, preprint

[12] Harer John L, Stability of the homology of the mapping class groups of orientable surfaces, Ann. Math. (2) 121 (1985) 215-249

[13] Harer John L, The virtual cohomological dimension of the mapping class group of an orientable surface, Invent. Math. 84 (1986) 157-176

[14] Hatcher Allen, Homological stability for automorphism groups of free groups, Comment. Math. Helv. 70 (1995) 39-62

[15] Hatcher Allen, Algebraic topology (Cambridge University Press) (2001)

[16] Hatcher Allen, On triangulations of surfaces, Topology Appl. 40 (1991) 189-194

[17] Hatcher Allen and Vogtmann Karen, Isoperimetric inequalities for automorphism groups of free groups, Pacific J. Math. 173 (1996) 425-441

[18] Hatcher Allen and Vogtmann Karen, The complex of free factors of a free group, Quart. J. Math. Oxford Ser. (2) 49 (1998) 459-468

[19] Hatcher Allen and Vogtmann Karen, Rational homology of Aut $\left(F_{n}\right)$, Math. Res. Lett. 5 (1998) 759-780

[20] Hatcher Allen and Vogtmann Karen, Homology stability for outer automorphism groups of free groups, Algebr. Geom. Topol. 4 (2004) 1253-1272

[21] Hatcher Allan, Vogtmann Karen and Wahl Natalie, Erratum to: Homology stability for outer automorphism groups of free groups, Algebr. Geom. Topol. 4 (2004) 1253-1272, by Hatcher and Vogtmann, Algebr. Geom. Topol. 6 (2006) 573-579

[22] Hatcher Allen and Wahl Nathalie, Stabilization for the automorphisms of free groups with boundaries, Geom. Topol. 9 (2005) 1295-1336 
[23] Hatcher Allen and Wahl Nathalie Erratum to: Stabilization for the automorphisms of free groups with boundaries, Geom. Topol. 9 (2005) 1295-1336, Geom. Topol. 12(2) (2008) 639-641

[24] Kim Y D, The Thurston boundary of Teichmüller space and curve complex, Topology Appl. 154(3) (2007) 675-682

[25] Laudenbach Francois, Topologie de la dimension trois: homotopie et isotopie (French) Astérisque, No. 12 (Paris: Société Mathématique de France) (1974)

[26] Luo Feng, Automorphisms of the complex of curves, Topology 39 (2000) 283-298

[27] Lyndon R C and Schupp P E, Combinatorial Group Theory (Berlin Heidelberg New York: Springer-Verlag) (1977)

[28] Luo Feng, A presentaion of the mapping class groups, arXiv.math.GT/9801025, v1, 7 Jan (1998)

[29] Luo Feng and Stong R Measured lamination spaces on surfaces and geometric intersection numbers, Topology Appl. 136(1-3) (2004) 205-217

[30] Masur H A and Minsky Yair, Geometry of the complex of curves I, Hyperbolicity, Invent. Math. 138 (1999) 103-149

[31] Scott Peter and Swarup Gadde A, Splittings of groups and intersection numbers, Geom. Topol. 4 (2000) 179-218 